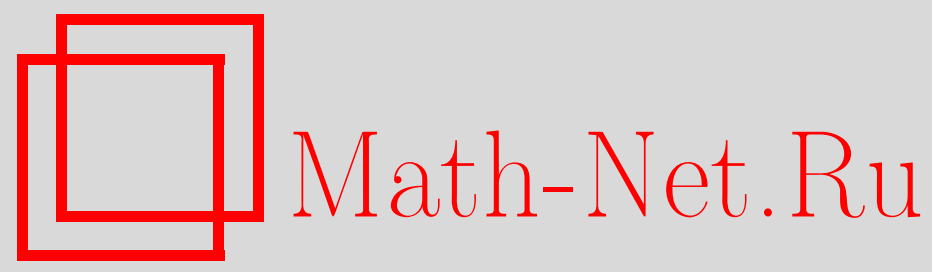

В. Б. Кудрявцев, И. С. Грунский, В. А. Козловский, Восстановление автоматов по фрагментам поведения, Дискрет. матем., 2009, том 21, выпуск 2, 3-42

DOI: https://doi.org/10.4213/dm1044

Использование Общероссийского математического портала Math-Net.Ru подразумевает, что вы прочитали и согласны с пользовательским соглашением http://www . mathnet.ru/rus/agreement

Параметры загрузки:

IP: 3.95 .254 .165

26 апреля 2023 г., 11:08:18 


\title{
Восстановление автоматов по фрагментам поведения
}

\author{
() 2009 г. В. Б. Кудрявцев, И. С. Грунский, В. А. Козловский
}

\begin{abstract}
Обзор содержит результаты, относящиеся к поведенческой и абстрактной теории конечных автоматов, являющиеся развитием содержащихся в предыдущем обзоре авторов результатов по теории представления автоматов с заданной точностью относительно различных классов и использования при их анализе идентификаторов состояний.
\end{abstract}

\section{1. Введение. Задачи, рассматриваемые в обзоре}

Данный обзор идейно связан с работой [58] и содержит результаты, являющиеся развитием результатов из [58] по теории представления автоматов с заданной точностью относительно различных классов и использования при их анализе идентификаторов состояний. Авторы сочли необходимым повторить во введении ряд положений указанной работы, а также приведенный в ней список литературы.

Результаты, составляющие содержание обзора, относятся к поведенческой и абстрактной теории конечных автоматов. Классическими задачами этой теории являются прямые задачи: анализ процессов преобразования информации, осуществляемых автоматами, и свойств автоматов, и обратные задачи: синтез автоматов с заданными свойствами и идентификации (восстановления, распознавания, расшифровки, контроля и диагностики) автомата путем экспериментов с ним.

Задача синтеза состоит в построении автомата по заданной его спецификации - заданию на необходимое, возможное и запрещенное поведение. Задача идентификации построение автомата путем проведения экспериментов с заданным "черным ящиком", являющимся реализацией этого автомата. В процессе эксперимента возникает фрагмент поведения автомата. Поэтому имеется единая исходная основа - фрагмент поведения, для решения задач анализа свойств автомата по его поведению и синтеза автомата, удовлетворяющего заданному поведению с некоторой точностью. Эти задачи и рассматриваются в работе. Описание поведения автомата задается дескриптором того или иного вида, позволяющим описать поведение автомата с заданной степенью точности.

Указанные задачи являются базовыми для двух научных направлений - формальных методов синтеза программно-аппаратных вычислительных систем и теории экспериментов с автоматами. В первом важную роль играют средства спецификации, и развитию этих средств уделяется большое внимание. Растет число языков спецификации, разрабатываются средства поддержки разработки спецификаций и методики их использования.

Экспериментом с автоматом называется процесс подачи на автомат последовательности входных сигналов, наблюдение соответствующего поведения автомата и вывод 
заключений о функционировании и свойствах автомата, основанных на этих наблюдениях и априорной информации об автомате. Основная задача теории экспериментов состоит в разработке эффективных экспериментов, позволяющих получить (распознать) определенные сведения о строении автомата, его функциях, о характеристиках процесса преобразования информации, осуществляемого этим автоматом. При этом возникает большой круг задач, связанных с классификацией экспериментов, с вопросами разрешимости задач распознавания тех или иных свойств автомата определенными видами экспериментов, с оценками сложности минимальных экспериментов, достаточных для решения тех или иных задач распознавания, а также с оценками сложности построения этих экспериментов. Теория экспериментов интенсивно разрабатывается, в ней получен ряд важных и принципиальных результатов [2-8]. Она имеет широкий круг применений и оказывает значительное влияние на развитие ряда математических и технических дисциплин (технической диагностики, теории динамических систем, теории формальных языков и грамматик, теоретического программирования и других).

Спецификация автомата и фрагменты поведения автомата являются примерами дескриптора автомата. При исследовании точности описания автомата фрагментами важную роль играют так называемые идентификаторы свойств автомата (состояний, входов, выходов) [6], являющиеся фрагментами, позволяющие однозначно идентифицировать эти свойства. Идентификаторы дают еще один пример дескриптора.

При решении задач синтеза и идентификации возникает необходимость описывать потенциально бесконечные классы автоматов финитными средствами. Одним из основных таких средств являются недетерминированные автоматы - еще один пример дескриптора.

Таким образом, при исследовании задач синтеза и идентификации автоматов возник и интенсивно используется ряд частных видов дескрипторов автомата, и эти дескрипторы изучаются своими особыми методами. В связи с этим возникает необходимость разработки теоретического осмысления и обобщения этих частных случаев с целью создания общих методов изучения, создания и использования дескрипторов автомата при решении задач синтеза и идентификации автомата.

Основными проблемами дескрипции автоматов являются анализ дескрипторов данного автомата, синтез автомата по заданным дескрипторам, представление автомата дескрипторами с заданной степенью точности.

Основное внимание в работе уделяется исследованию последней из них. В качестве основных модельных дескрипторов выступают фрагменты автомата. Это объясняется следующими соображениями.

Проблематика восстановления автомата по заданному фрагменту возникла в теории экспериментов и технической диагностике, когда фрагмент - это тест и реакция на него, полученная в эксперименте, а также при синтезе, когда фрагмент - это задание на функционирование синтезируемого автомата. Эта проблематика состоит в анализе точности описания автомата фрагментами, априорно заданными или полученными в эксперименте; создании методов построения фрагментов, представляющих автомат с заданной степенью точности; создании методов синтеза автомата по фрагментам. Указанная проблематика развивается в рамках четырех направлений: синтеза управляющих и вычислительных систем, технической диагностики таких систем, синтаксического распознавания образов, теории экспериментов с автоматами. Развитие этих направлений происходит достаточно независимо друг от друга под определяющим влиянием теории экспериментов.

Основание теории экспериментов с автоматами положено Э. Муром, исследования которого затрагивали два взаимосвязанных аспекта: неотличимость автоматов никакими кратными и никакими простыми (однократными) экспериментами; распознавание автоматов и их состояний с помощью таких экспериментов. Становление теории экспериментов 
тесно связано с работами А. М. Богомолова и его учеников, М. П. Василевского, А. Гилла, В. Н. Носкова, Н. В. Евтушенко, А. Ф. Петренко, И. К. Рысцова, В. А. Твердохлебова, С. В. Яблонского, Ф. Хенни и его многочисленных последователей, П. Штарке и многих других. Эти исследования можно разделить на три этапа: комбинаторный, сложностной и характеризационный.

Комбинаторный этап касался построения средств анализа автоматов и алгоритмов проведения экспериментов с ними, исходя из комбинаторно-мощностных соображений. Методы и результаты этого этапа достаточно полно представлены в книге А. Гилла [12].

На сложностном этапе основное внимание уделялось построению оптимальных по сложности алгоритмов и нахождению сложности проведения экспериментов, а также оптимальных по сложности методов анализа поведения автомата. Это потребовало анализа структуры экспериментов. Этот этап инициирован работами Ф. Хенни и М. П. Василевского, которые проводили построение контрольных экспериментов путем помещения во входные последовательности специальных подпоследовательностей (диагностических, установочных, локализующих, характеристических и т. п.), по реакции на которые исследуемого автомата можно идентифицировать его внутренние состояния. На этом этапе исследовались различные виды таких последовательностей и способы их размещения в эксперименте. Методы и результаты второго этапа достаточно полно представлены в книге В. Б. Кудрявцева, С. В. Алешина, А. С. Подколзина [7]. Один из авторов настоящего обзора (И. С. Грунский), по-видимому, первым назвал эти последовательности и реакции на них идентификаторами состояний, начал их систематическое изучение [4] и обратил внимание на существование идентификаторов других ненаблюдаемых компонент поведения (например, входных последовательностей [6]). На втором этапе получено большое количество частных способов построения контрольных и распознающих экспериментов для случаев, когда они заведомо существуют. Неисследованными остались условия существования и структура таких экспериментов в общем случае. Очень трудным оказался вопрос: что должно присутствовать в этих экспериментах, а что является издержками алгоритмов их построения, то есть вопрос характеризации вышеуказанных экспериментов. Появление первых характеризационных теорем [47] можно отнести уже к становлению третьего, характеризационного, этапа.

Как упоминалось выше, в последнее время возникло важное и интенсивно развивающееся направление “формальные методы синтеза компьютерных систем”, во многом объединяющее вышеуказанные направления. Для него основными задачами являются задачи контроля таких систем на всех этапах их жизни: проектирования, изготовления и эксплуатации (контроль и диагностика). Появилось значительное число средств описания таких систем на различных уровнях (эксперименты, анкетные языки, $k$-наборы, частичные и недетерминированные автоматы, временные логики и т.п.). Эти средства фактически описывают фрагменты поведения синтезируемого автомата, зачастую порождаемые неклассическим способом фиксации его поведения. При этом каждый вид таких средств изучается своими специальными методами в отрыве от остальных. Такое разнообразие требует создания общего понятия фрагмента, исследования условий существования, структуры, сложности фрагментов, позволяющих описывать, восстанавливать, идентифицировать автомат с заданной точностью, а также разработки методов создания таких фрагментов. Исследование этого комплекса задач составляет, на наш взгляд, содержание третьего этап - характеризационного. Актуальность исследований в этом направлении, в силу вышесказанного, все возрастает. Этим исследованиям, выполненным в значительной степени авторами обзора либо под их руководством, посвящена значительная часть работы. 
Базовым понятием, на которое опирается изучение вышеуказанного круга задач, является предложенное понятие фрагмента, являющееся естественным обобщением большого числа известных фрагментов частного вида и обладающее их характерными свойствами. Другое ключевое понятие - понятие идентификатора ненаблюдаемых компонент функционирования автомата, то есть фрагмента, позволяющего однозначно определить значения этих компонент. Ряд принципиальных результатов по теории фрагментов приведен в [6].

Введено общее понятие представления автомата-эталона с заданной точностью (подобием) относительно априорного класса автоматов как пары (фрагмент, кофрагмент) автомата-эталона, которая может быть такой парой для автомата из априорного класса только тогда, когда он подобен эталону. Это понятие охватывает и обобщает ряд частных понятий (контрольные, распознающие эксперименты, анкетные языки, $k$-наборы и т. п.), известных в теории автоматов. Исследована структура текстуальных представлений, то есть представлений, состоящих только из фрагмента автомата и не содержащих кофрагмента. Эта структура описана в терминах наличия и расположения в представлении идентификаторов состояний эталона. Для этого введена операция замыкания фрагмента по так называемым верифицированным идентификаторам эталона, и структура текстуальных представлений описана в терминах этого замыкания. Из этих результатов следует, в частности, обоснование методов построения контрольных экспериментов, предложенных Ф. Хенни, М. П. Василевским и рядом их последователей. Найдены условия, при которых фрагмент является представлением точно тогда, когда его замыкание по соответствующим идентификаторам изоморфно эталону. Тем самым выделены случаи, когда идентификаторы состояний в представлениях необходимы. Найден ряд критериев (в терминах замыканий), при которых фрагмент является представлением в случае, когда априорный класс конечен и состоит из автоматов, число состояний которых не превосходит числа состояний эталона, а сам эталон обладает дополнительными свойствами, важными в приложениях теории автоматов. Ряд этих критериев был получен с единых позиций сведением задачи анализа фрагментов с целью выявления свойства “быть представлением” к задаче анализа раскрасок некоторых графов. Приведены результаты по оценке сложности задачи распознавания представлений автоматов. С использованием полученных характеризационных теорем доказана $N P$-полнота указанной задачи распознавания для ряда случаев. Кроме того, получены неулучшаемые оценки длин контрольных экспериментов, определяющие предельные нижние границы этих длин для экспериментов с любым эталоном относительно рассмотренных классов автоматов.

Как указывалось выше, в последнее время в прикладных задачах возникает необходимость рассматривать потенциально бесконечные классы, заданные финитными дескрипторами. Рассмотрены два типа таких дескрипторов: недетерминированные автоматы и маркеры-идентификаторы состояний специального вида. Введены два вида финитноопределенных классов, соответствующие этим дескрипторам. Исследуется структура этих классов. Особое внимание уделяется свойствам контрольных и распознающих экспериментов относительно этих классов.

Тематика, отраженная в обзоре, интенсивно развивается, как в направлении решения математических вопросов, так и применения в прикладных исследованиях, связанных с синтезом, тестированием и верификацией сложных программно-аппаратных систем. Ряд результатов является окончательными, однако они являются лишь очередным шагом в исследовании задач анализа и синтеза автоматов по их поведению, которые постоянно наполняются новым содержанием и требуют дополнительных усилий и средств их разрешения. 


\section{2. Структура представлений автоматов}

Проблема представления автомата фрагментами рассматривается с двух точек зрения. Первая из них - эксперименты с автоматами. Постановка задачи в этом случае такова. Задан класс $F$ автоматов, в котором выделен “исправный” автомат-эталон $A$. Остальные автоматы из $F$ называются “неисправностями” эталона. Кроме этого, предъявлен черный ящик $B$, о котором известно, что он принадлежит классу $F$. Требуется найти такое множество $P$ входных слов (тестовых последовательностей), по реакции на которые автомата $B$ можно определить:

(a) совпадает ли $B$ с эталоном (контрольный эксперимент);

(б) функции автомата $B$ (распознающий эксперимент).

В этом случае тест $P$ вместе с соответствующими реакциями автомата $B$ является искомым фрагментом поведения. Известен ряд работ, в которых предложены различные алгоритмы построения вышеуказанных экспериментов и найдены оценки их сложности [3-7]. В последнее время появился ряд неклассических видов экспериментов с более сложными степенями точности, определяемыми приложениями (сертификацией протоколов в сетях ЭВМ [27-28], тестированием компоненты сети автоматов [29] и т. п.) Вторая точка зрения связана с описанием исходного задания автомата при его синтезе. При этом автомат задается в виде системы вход-выходных слов, которая представляет информацию двоякого вида: то, что должен реализовывать автомат (предписанное поведение) и то, что автомат не должен реализовывать (запрещенное поведение) $[30,31]$. Известен ряд вариантов такого описания: анкетные языки [30], $k$-наборы [20], языки эквивалентных преобразований [31]. Предложены алгоритмы получения таких описаний по заданному автомату и алгоритмы синтеза автомата по заданным описаниям.

Анализ полученных результатов показывает, что до сих пор мало изученными являются следующие вопросы.

(1) Какова та граничная информация об автомате и классе, относительно которого описывается автомат, при которой вышеуказанное описание того или иного вида (эксперимент, анкетный язык) существует, а без которой не существует?

(2) Какова структура вышеуказанного описания автомата, что обязательно должно присутствовать в описании, а что является издержками алгоритма его получения?

(3) Какова сложность описаний, сложность их построения и сложность восстановления автомата по его описанию?

Эти вопросы являются актуальными не только для теории автоматов, но и для смежных дисциплин таких, как техническая диагностика [32,33], идентификация систем управления [34] и другие.

Введем необходимые определения. Класс всюду определенных приведенных автоматов в алфавитах $X, Y$ обозначим $\mathbf{A}(U)$, где $U=X \times Y$. Пусть $F \subseteq \mathbf{A}(X, Y)$, и $A=(S, X, Y, \delta, \lambda)$ - некоторый автомат, называемый эталоном. Пусть также $\tau-$ бинарное отношение подобия на классе $\mathbf{A}(X, Y)$. Если $(A, B) \in \tau$, то автомат $B$ назовем подобным эталону. Обычно $A \in F$.

Напомним определения фрагмента и представления [58]. Пусть $v_{i}=U_{i 1} U_{i 2} \ldots U_{i k}-$ некоторые слова в алфавите $2^{U}, i=1,2$, то есть $U_{i j} \subseteq U$ для всех $i, j$. Если $U_{1 j} \subseteq U_{2 j}$ для всех $j, 1 \leqslant j \leqslant k$, то первое слово $v_{1}$ назовем уточнением второго слова $v_{2}$, а 
второе - расширением первого, что обозначим включением $v_{1} \subseteq v_{2}$. Если слово $v_{i}$ понимать как терм в алгебре Клини, определяющий язык в алфавите $U$, то есть считать, что $v_{i}=\left\{u_{1} \ldots u_{k} \mid u_{j} \in U_{i k}\right\}$, то последнее включение совпадает с обычным теоретикомножественным включением. Введенное включение распространяется на языки в алфавите $2^{U}$. Пусть $V_{i}-$ множество слов в этом алфавите, $i=1,2$. Если для каждого $v_{1} \in V_{1}$ найдется его расширение $v_{2} \in V_{2}$, то $V_{1}$ назовем уточнением $V_{2}$, а $V_{2}$ - расширением, что обозначим $V_{1} \subseteq V_{2}$. Если $V_{i}$ понимать как объединение языков $v \in V_{i}$, то последнее включение не противоречит теоретико-множественному включению.

Пусть $R_{i}=\left(T_{i}, 2^{U}, \Delta_{i}\right)$ - автоматы Медведева, $i=1,2$. Пусть $\varphi: T_{1} \rightarrow T_{2}$ - отображение, для которого, если $\left(t_{1}, U_{1}, t_{2}\right)$ - дуга автомата $R_{1}$, то найдется такое уточнение $U_{2} \subseteq U_{1}$, что $\left(\varphi\left(t_{1}\right), U_{2}, \varphi\left(t_{2}\right)\right)$ - дуга автомата $R_{2}$. В отличие от обычного гомоморфизма, отображение $\varphi$ будем называть слабым гомоморфизмом. Факт существования слабого гомоморфизма будем записывать в виде $R_{1} \sqsubseteq R_{2}$.

Пусть $R, Q-$ возможно бесконечные автоматы Медведева в алфавите $2^{U}$. Пусть $A$ - некоторый автомат в алфавите $U$. Автомат $R$ назовем фрагментом автомата $A$, если $R \sqsubseteq A$. Фрагмент $R$ назовем непосредственным, если метка каждой его дуги состоит из одной буквы внешнего алфавита $U$ и $R$ является детерминированным автоматом. В противном случае фрагмент называется косвенным. Назовем $Q$ кофрагментом автомата $A \in \mathbf{A}(X, Y)$, если каждая компонента связности $V \subseteq Q$ не является фрагментом автомата $A$. Через $\mathbf{A}(R, Q)$ обозначим класс всех автоматов из $\mathbf{A}(X, Y)$, для которых $R$ является фрагментом, а $Q-$ кофрагментом. Ясно, что $\mathbf{A}(R, Q)=\mathbf{A}(R)-(\bigcup \mathbf{A}(V))$, где объединение выполняется по всем компонентам связности $V$ кофрагмента $Q$

Пару $\langle R, Q\rangle$ назовем представлением эталона $A$ относительно $F$ с точностью $\tau$, если одновременно выполняются следующие условия:

(1) $R$ является фрагментом, а $Q-$ кофрагментом эталона;

(2) для любого $B \in F$, если $R$ является фрагментом, а $Q-$ кофрагментом автомата $B$, то $B \in \tau(A)$.

Класс всех представлений эталона относительно $A$ и $\tau$ обозначим $R(A, F, \tau)$. Представление назовем текстуальным в случае, когда $Q$ пусто, и информаторным в противном случае. Класс всех текстуальных представлений обозначим $R_{T}(A, F, \tau)$.

Из определения представлений эталона следует, что $(R, Q) \in R(A, F, \tau)$ точно тогда, когда одновременно выполняются условия

(1) $A \in \mathbf{A}(R, Q) \cap F$,

(2) $\mathbf{A}(R, Q) \cap F \subseteq \tau(A)$.

Пусть $Q=\sum_{i} Q_{i}, i \in I,-$ разложение кофрагмента в прямую сумму его $F$ компонент связности. Тогда эти условия равносильны соотношениям

(1) $A \in F \cap \mathbf{A}(R) \cap\left(\bigcap_{i} \overline{\mathbf{A}}\left(Q_{i}\right)\right)$,

(2) $\left(F \cap \mathbf{A}(R) \cap\left(\bigcap_{i} \overline{\mathbf{A}}\left(Q_{i}\right)\right) \subseteq \tau(A)\right.$.

Здесь $\overline{\mathbf{A}}\left(Q_{i}\right)$ - дополнение класса $\mathbf{A}\left(Q_{i}\right)$ до $\mathbf{A}(X, Y)$.

Если выходной алфавит $Y$ содержит только один символ, то $\mathbf{A}(X, Y)=\{A\}$, и класс представлений совпадает с классом всех фрагментов эталона. Поэтому в дальнейшем, если не оговорено противное, считаем, что $|Y| \geqslant 2$.

Рассмотрим примеры представлений. 
1. Пусть $F$ состоит из всех автоматов с числом состояний, не превосходящим числа состояний эталона, то есть, как говорят в технической диагностике, неисправность не увеличивает числа состояний автомата [33]. Пусть отношение $\tau$ является отношением равенства (изоморфизма) автоматов. Пусть $p$ - контрольная в смысле Хенни [35] последовательность для $A$. Это значит, что если исследуемый “черный ящик” $B$, принадлежащий $F$, отреагировал на слово $p$ словом $q$, для которого $(p, q)$ может быть порождено эталоном, то $A=B$. Тогда строчный автомат $R(p, q)$ является представлением.

Следуя [35], можно выбрать $F$ равным классу всех автоматов, число состояний которых не превосходит удвоенного числа состояний эталона и считать, что $B$ подобен $A$, если $A \subseteq B$, то есть если $B$ является реализацией $A$. Такое $\tau$ соответствует проверке работоспособности автомата [33], и в этом случае для контрольного эксперимента $(p, q)$ автомат $R(p, q)$ является представлением.

2. В [36] рассматривается проблема контроля (обнаружения) неисправностей, если классом $F$ является класс всех автоматов, число состояний которых не превосходит заданного натурального числа. Этот класс содержит в себе большинство естественно определяемых классов неисправностей. Предложен алгоритм построения такого множества $P=\left\{p_{i}\right\}, 1 \leqslant i \leqslant k$, входных слов, что если “черный ящик” $B$ в состоянии $t$ порождает эксперимент $Q=\left\{\left(p_{i}, q_{i}\right)\right\}, i \in\{1, \ldots, k\}$, для которого $Q \subseteq \lambda_{A s}$, то $\lambda_{A s}=\lambda_{B t}$. Таким образом, древовидный фрагмент $D(Q)$, полученный из $\sum_{i} R\left(p_{i}, q_{i}\right)$, является представлением эталона относительно вышеуказанного $F$ и $\tau$, определяемого соотношением $(A, B) \in \tau$, если $\lambda_{A s}=\lambda_{B t}$, где $s$ и $t$ начальные состояния автоматов.

В силу сказанного, можно считать, что кратные и простые контрольные эксперименты являются частным видом представлений. Аналогичные рассуждения можно провести для распознающих экспериментов [3-8] и анкетных языков [30].

3. Рассмотрим $k$-набор - конечное множество вход-выходных слов вида $(p, q)=$ $\left(x_{1}, y_{1}\right) \ldots\left(x_{i}, y_{i}\right) *\left(x_{i-1}, y_{i-1}\right) \ldots\left(x_{k}, y_{k}\right)$, описывающий сильно связные автоматы с точностью до $\tau=v_{1}$. Звездочка показывает, что начальный отрезок слова $(p, q)$, стоящий слева от нее, оканчивается в том же состоянии автомата, в котором оканчивается все слово. Если по слову $(p, q)$ построить $R(p, q)$, отождествить в нем состояния $i+1$ и $k+1$ и провести такое построение для каждого слова из $k$-набора, то получим представление $R$ сильно связного эталона $A$ относительно бесконечного класса сильно связных автоматов.

4. В [4] предложена методика анализа вход-выходных слов с помощью идентификаторов состояний. В результате этого анализа получается так называемый предельный автомат, который в случае, если анализировался контрольный или распознающий эксперимент, является представлением автомата $A$ относительно заданных $F$ и $\tau$.

5. В [37] рассмотрен случай, возникающий при сертификации протоколов [28] и контроле компоненты сети автоматов [29], когда класс А исправных автоматов задан в виде класса реализаций недетерминированного автомата $A$, а неисправных $\mathbf{B}-$ в виде класса реализаций недетерминированного автомата $B$. В этой работе строится эксперимент, определяющий, является “черный ящик” реализацией автомата $A$ или реализацией автомата $B$. В процессе проведения этого эксперимента получается множество $W$ вход-выходных слов, порожденных “черным ящиком”. Автомат $R(W)$ является текстуальным представлением "черного ящика", а точность определяется разбиением $\{A, B\}$. Заметим, что в этом случае эти классы могут быть как конечными так и бесконечными.

Рассмотренные примеры показывают важность и актуальность исследования проблемы представления фрагментами. В [6, 22] заложены основы такого исследования. В [58] были изложены условия существования представлений различного вида при различных 
предположениях на свойства эталона, класса $F$ и точности представления $\tau$. В данном разделе основное внимание уделено изучению структуры представлений в случае, когда представлением является только фрагмент $R$ эталона, а также сложности представлений, как метрической, так и их распознавания. Эти задачи рассматривались в первую очередь для определенно диагностируемых порядка $k$ автоматов. Такие автоматы интенсивно изучались в теории экспериментов с автоматами $[4,6,12,15,16]$ и часто встречаются в прикладных исследованиях. В разделе приведены необходимые и достаточные условия, при которых фрагмент является представлением определенно диагностируемого порядка 1 эталона относительно класса $F_{n}$ всех автоматов с числом состояний, не превосходящим число $n$ состояний эталона. Для случая $\tau=\varepsilon$ (то есть изоморфизма автоматов) описана структура минимальных представлений. Получены необходимые и достаточные условия быть представлением такого же эталона относительно класса автоматов, порожденных из эталона локальными преобразованиями последнего, и $\tau$, являющихся отношениями неотличимости различного вида. И, наконец, найден критерий, при котором вход-выходное слово является контрольным экспериментом для определенно диагностируемого порядка $k, 1 \leqslant k \leqslant 11$, эталона относительно $F_{n}$ и $\tau=\varepsilon$.

В основном, изучается структура текстуальных правильных представлений с точки зрения наличия и расположения в них идентификаторов состояний эталона. При этом в разделе рассматриваются только правильные фрагменты, то есть, конечные, детерминированные, в общем случае частичные автоматы во внешнем алфавите $U=X \times Y$.

Использование идентификаторов состояний таких, как диагностические и установочные эксперименты при построении контрольных и распознающих экспериментов проводилось с самого начала развития теории экспериментов в работах Э. Мура, А. Гилла, Ф. Хенни, М. П. Василевского и др. $[3,7,12,14,15,16,35,36]$. При этом идентификаторы состояний эталона специальным образом помещаются в контрольные и диагностические эксперименты, и поэтому эти эксперименты тоже являются идентификаторами состояний эталона.

В [6] показано, что имеет место следующее утверждение.

Предложение 2.1. Для всякого класса $F$, отличимого от эталона $A$ по $\tau$, существует такое древовидное представление для $(A, F, \tau)$, которое, при соответствующем выделении его состояний, является идентификатором каждого состояния эталона.

Следующие результаты показывают, насколько идентификаторы полезны и необходимы при построении представлений для $(A, F, \tau)$.

Пусть задан некоторый автомат $B$. Фрагмент $R, R \leqslant B$, с выделенным состоянием $t$ называется идентификатором состояния $s$ этого автомата, если для любого гомоморфизма $\varphi$ фрагмента $R$ в $B$ выполняется равенство $\varphi(t)=s$. На множестве $J_{B}$ всех идентификаторов состояний автомата $B$ введем отношение совместимости $\sigma_{B}$ : два идентификатора совместимы, то есть принадлежат $\sigma_{B}$, если они являются идентификаторами одного и того же состояния автомата $B$. Ясно, что $\sigma_{B}$ является эквивалентностью на множестве $J_{B}$.

Пусть заданы некоторый класс $F \in \mathbf{A}(U)$ и эталон $A \in \mathbf{A}(U)$. Пусть $R-$ некоторый (правильный) фрагмент эталона. Идентификатор $I$ состояний эталона назовем верифицированным во фрагменте $R$, если $I$ является идентификатором состояний каждого такого автомата $B \in F$, что $R \leqslant B$. Через $J_{R}$ обозначим класс всех верифицированных в $R$ идентификаторов состояний эталона, а через $\sigma_{R}$ - отношение совместимости на $J_{R}$, причем $\left(I_{1}, I_{2}\right) \in \sigma_{R}$, если $\left(I_{1}, I_{2}\right) \in \sigma_{B}$ для всех автоматов $B \in F$. Очевидно, что $\sigma_{R}-$ эквивалентность на $J_{R}$. Система $(J, \sigma)$, где $J \subseteq J_{R}$ и $\sigma-$ некоторая эквивалентность на $J$, для которой $\sigma \subseteq \sigma_{R}$, будет называться верифицированной в $R$ системой идентификаторов. Эквивалентность $\sigma$, содержащая только пары $(I, I)$, где $I \in J$, называется диагональю 
и является наименьшей по включению эквивалентностью на $J$. Иногда диагональ $\sigma$ будем называть пустым отношением совместимости идентификаторов, так как такое $\sigma$ не содержит информации о совместимости разных идентификаторов из $J$.

Пусть $(J, \sigma)$ - некоторая верифицированная в $R$ система идентификаторов состояний. Эта система определяет на множестве $T$ состояний фрагмента $R$ отношение $\beta:\left(t_{1}, t_{2}\right) \in \beta$, если найдется такая пара идентификаторов состояний $\left(I_{1}, I_{2}\right) \in \sigma_{R}$, для которых $t_{i}-$ это выделенное состояние идентификатора состояний, $i=1,2$ и $I_{i} \leqslant R$. Другими словами, $\left(t_{1}, t_{2}\right) \in \beta$, если в $R$ содержатся идентификаторы состояний $t_{i}$ и гомоморфные прообразы этих идентификаторов совместимы по $\sigma_{R}$. Отношение $\beta$ является рефлексивным и симметричным. Наименьшая (по включению $\subseteq$ ) эквивалентность $\alpha$, для которой $\beta \subseteq \alpha$, называется транзитивным замыканием $\beta$. Конгруэнтное замыкание $\alpha$ однозначно определяет фрагмент $R_{1}$, для которого $R \leqslant R_{1}$, по следующему правилу. Отождествляем состояния фрагмента $R$, принадлежащие одному классу эквивалентности $\alpha$. Получим в общем случае недетерминированный частичный автомат $R^{\prime}$, у которого множество состояний равно $T / \alpha$. Пусть $\left(t_{1}, t_{2}\right) \in \alpha$. По определению верифицированной системы $(J, \sigma)$, это означает, что для любого гомоморфизма $\varphi$ фрагмента $R$ в эталон выполняется равенство $\varphi\left(t_{1}\right)=\varphi\left(t_{2}\right)$. Следовательно, $\lambda_{R t_{1}} \cup \lambda_{R t_{2}} \subseteq \lambda_{A s}$, где $\varphi\left(t_{1}\right)=s$. Проведем детерминизацию автомата $R^{\prime}$ : если в нем содержатся дуги $\left(t, x, y_{1}, t_{1}\right)$ и $\left(t, x, y_{2}, t_{2}\right)$, где $t_{1} \neq t_{2}$, то в силу последнего включения $y_{1}=y_{2}$, и состояния $t_{1}, t_{2}$ отождествляем. Проводя такое отождествление до тех пор, пока это возможно, получим, в силу сказанного, частичный детерминированный автомат $R_{1}$, для которого $R \leqslant R_{1}$. На множестве состояний автомата $R_{1}$ опять определяем отношение $\alpha$, затем строим $R_{1}$ и, детерминизируя его, получим $R_{2}$. Проводя такие построения, получим последовательность автоматов $R \leqslant R_{1} \leqslant R_{2} \leqslant \ldots \leqslant R_{i} \leqslant \ldots$ По построению все $R_{i}$ являются фрагментами эталона, и число состояний автомата $R_{i}$ не меньше числа состояний автомата $R_{i+1}$. Поэтому найдется такое $i$, что $R_{i}=R_{i+1}$. В этом случае отношение $\alpha$ на состояниях автомата $R$ является тривиальным, то есть $\left(t_{1}, t_{2}\right) \in \alpha$, если $t_{1}=t_{2}$. Автомат $R_{i}$ в этом случае назовем замыканием автомата $R$ по верифицированной системе $(J, \sigma)$ идентификаторов состояний.

Пусть $Q-$ замыкание фрагмента $R$ по некоторой верифицированной системе $(J, \sigma)$. Процедура построения $Q$ однозначно определяет канонический гомоморфизм $\varphi$ автомата $R$ на $Q$, для которого $\varphi(R)=Q$. Ядро гомоморфизма $\varphi-$ это такая конгруэнция $\alpha$, что $R / \alpha=Q$. Из неравенства $R \leqslant Q$ следует включение $\mathbf{A}(R) \supseteq \mathbf{A}(Q)$. Легко показать, что обратное включение не имеет места. Пусть $\mathbf{F}(R)=\mathbf{A}(R) \cap F$. Справедливо следующее полезное утверждение.

Лемма 2.1. Для любой верифицированной системы $(J, \sigma)$ фрагмента $R$ класс $\mathbf{F}(R)$ совпадает с классом $\mathbf{F}(Q)$, где $Q$ - это замыкание фрагмента $R$ по этой системе.

В следующем разделе верифицированные системы будут рассмотрены в более общей постановке.

Из этой леммы очевидно выводятся следующие полезные утверждения. Пусть задана тройка $(A, F, \tau), R_{i}$ - правильные фрагменты эталона, $i=1,2$, и $Q_{i}$ - замыкание фрагмента $R_{i}$ по некоторой верифицированной в $R_{i}$ системе $\left(J_{i}, \sigma_{i}\right)$.

Следствие 2.1. Если $A \subseteq Q_{1} / \varepsilon$, то $R_{1}-$ представление эталона $A$ относительно $F$ $u \tau$.

Если $Q_{1}=Q_{2}$, то $R_{1}-$ представление для $(A, F, \tau)$ точно тогда, когда $R_{2}-$ тоже представление. 
Первое утверждение следствия 2.1 в неявном виде использовалось в ряде работ, начиная с работы Ф. Хенни [35], при построении контрольных экспериментов, причем, как правило, стремились к тому, чтобы $Q_{1}$ совпадало с $A$. В роли верифицированной системы выступало чаще всего множество $\{(p, \lambda(s, p))\}_{s \in S}$ простых начальных идентификаторов эталона, порожденное диагностическим словом $p$.

Условие первого утверждения следствия 2.1 является только достаточным в общем случае. Однако в некоторых случаях оно является и необходимым. Рассмотрим эти случаи.

Пусть $F=\varepsilon_{i}(A), \tau=\iota$ и $D_{A}^{i}=\sum_{s \in S} D\left(\lambda_{s}^{i}\right)$. Заметим, что в этом случае $F \subseteq \mathbf{A}\left(D_{A}^{i}\right)$.

Теорема 2.1. $D_{A}^{i}$ является представлением эталона относительно $\varepsilon_{\iota}(A)$ и ८ тогда $u$ только тогда, когда замыкание фрагмента $D_{A}^{i}$ по некоторой системе $(J, \sigma)$, верифицированной в $R$, изоморфно эталону.

Теорема 2.1 дает первый пример ситуации, когда при построении представлений идентификаторы необходимы. Эта теорема является теоремой существования и не указывает, каким образом строить представление.

Рассмотрим структуру представлений в случае, когда $F-$ это $n$-полный класс $F_{n}$, где $n=n_{A}$, а $\tau=\iota$. Пусть $R \leqslant A$. Дуга $\left(t, x, y, t^{\prime}\right)$ автомата $R$ называется критической по гомоморфизму $\varphi$ этого фрагмента в эталон, если она является единственным прообразом по $\varphi$ некоторой дуги эталона. Состояние $t$ называется висячим, если $\delta_{R}(t, x)$ не определено ни для какого $x$.

Состояние автомата $R$ называется изолированным, если оно является висячим и преходящим одновременно. Дуга $\left(t, x, y, t^{\prime}\right)$, где $t \neq t^{\prime}$ называется изолированной, если она составляет компоненту связности в графе $R$. Представление $R$ назовем тупиковым, если, удаляя из него хоть одну дугу, получим автомат, не являющийся представлением.

Слово $\left(x_{1}, y_{1}\right) \ldots\left(x_{i}, y_{i}\right)$ называется неприводимым обходом (по всем дугам) эталона, если оно является обходом эталона, начиная из некоторого состояния $s$, в то время как $\left(x_{1}, y_{1}\right) \ldots\left(x_{i-1}, y_{i-1}\right)$ обходом из этого состояния не является. Через $d(w)$ будем обозначать длину вход-выходного слова $w$.

Рассмотрим случай, когда эталон является автономным автоматом, то есть когда $X$ состоит из единственного символа. Легко видеть, что всякий приведенный автономный автомат является определенно диагностируемым автоматом порядка не большего $n-1$.

Теорема 2.2. Для автономного эталона равносильны следующие утверждения:

(1) $w \in \Phi_{A}$ является контрольным экспериментом относительно $F_{n} u \iota$;

(2) $w=w^{\prime} w^{\prime \prime}$, где $w^{\prime}-$ неприводимый обход, а $w^{\prime \prime}-$ начальныий идентификатор некоторого состояния эталона;

(3) замыкание $Q$ фрагмента $R(w)$ по $(J, \sigma)$, где $J$ cocmouт из простых начальных идентификаторов, верифицированных в $R(w)$, а $\sigma$-диагональ, изоморфно эталоHy.

Второе утверждение теоремы 2.2 дает конструктивный способ проверки, является ли предъявленное вход-выходное слово контрольным экспериментом для эталона относительно $F_{n}$ и $\iota$. Это утверждение дает также алгоритм построения такого эксперимента. Из теоремы 2.2 непосредственно вытекает, что слово $x^{2 N-1}$, поданное на вход автономного автомата с не более, чем $N$ состояниями, вместе с реакцией $q$ исследуемого автомата на это слово позволяет распознать исследуемый автомат.

Результаты последней теоремы позволяют оценить длину контрольного эксперимента, указанного в ней. 
Следствие 2.2. Длина кратчайшего контрольного эксперимента для автономного автомата относительно $F_{n}$ и เ не может быть меньше $n+1$ и не превосходит $2 n-1$, причем для всех $n$ эти оценки достижимы.

Рассмотрим структуру представлений для частных видов эталона. Пусть дан некоторый автомат. Его подавтомат, порожденный всеми такими состояниями, в которых оканчивается хоть одно вход-выходное слово длины $k$, назовем $k$-м ядром этого автомата. Известно, что если автомат является автоматом с конечной памятью порядка $k$, то его $k$-е ядро включается в конечный фактор.

Теорема 2.3. Пусть эталон сильно связен или является автоматом с конечной памятью порядка $k$, и класс представлений эталона относительно некоторого $F$ u $v_{1}$ не пуст. Eсли для фрагмента $R$ эталона и некоторой верифицированной системь $(J, \sigma) k$-е ядра эталона и замыкания $Q$ фрагмента $R$ по этой системе изоморфны, а $\Phi_{A}^{k}=\Phi_{Q}^{k}$, то $R$ - представление относительно $F u v_{1}$.

Пусть $F-n$-полный класс $F_{n}, \tau=v_{1}$, и класс $R_{T}\left(A, F_{n}, v_{1}\right)$ не пуст.

Зафиксируем некоторый фрагмент $R$ эталона и верифицированную в $R$ систему $(J, \sigma)$, у которой $J$ состоит из всех верифицированных в $R$ простых начальных и конечных идентификаторов длины, не большей $k$, а $\sigma-$ сужение $\sigma_{R}$ на $J$. Пусть $Q-$ замыкание $R$ по $(J, \sigma)$.

Теорема 2.4. Пусть $A$ - определенно диагностируемый автомат порядка $k$ и $\Phi_{Q}^{k}=\Phi_{A}^{k}$. $R$ является представлением эталона относительно $F_{n} u v_{1}$ тогда и только тогда, когда $k$-е ядра эталона и замыкания $Q$ изоморфны.

Эта и предыдущие теоремы являются теоремами существования, так как не дают метода построения представлений. Центральным моментом этих результатов является нахождение верифицированной в $R$ системы идентификаторов состояний. Некоторые частные приемы верификации идентификаторов рассмотрены в [4]. Общие методы верификации, по-видимому, очень сложны и до сих пор не разработаны. Поэтому представляет интерес рассмотрение альтернативных подходов к изучению представлений, где верификация заменена проверкой некоторых конструктивных свойств. Один из возможных подходов рассмотрен ниже.

Рассмотрим условия, при которых правильный фрагмент $R$ относительно $F_{n}$ и $\iota$ является представлением для эталона $A$ в предположении, что он является определенно диагностируемым автоматом порядка $k$.

Пусть $\varphi$ - гомоморфизм автомата $R$ на $A$. Каждому состоянию $s$ эталона поставим в соответствие семейство $v_{s}$ подмножеств входных слов длины $k$ по правилу $z \in v_{s}$, если в $R$ существует состояние $t \in \varphi^{-1}(s)$ такое, что $z$ состоит из всех слов $p$ длины $k$, для которых $\delta_{R}(t, p)$ определено. Семейство $v_{s}$ назовем правильным, если элементы $v_{s}$ не сравнимы по включению. Семейство $v_{s}$ назовем полным по $X$, если для всякого $x \in X$ найдется класс $z \in v_{s}$, содержащий слово, начинающееся символом $x$. Положим $M(A, \varphi)=\left\{v_{s}\right\}_{s \in S}$. Через $M(A, \varphi)$ обозначим семейство, состоящее из всех элементов $z$ всех семейств $v_{s} \in M(A, \varphi)$. Семейство $M(A, \varphi)$ назовем простым, если для любого $B \in F_{n}$ и любого гомоморфизма $\psi$ автомата $R$ на $B$ из равенства $\bigcup M(A, \varphi)=\bigcup M(B, \psi)$ следует равенство $M(A, \varphi)=M(B, \psi)$. Введем операцию сложения семейств $v_{s}$ следующим образом: слова $p, q \in X^{k}$ принадлежат одному классу семейства $\left[v_{a}+v_{b}\right]$, если существует последовательность $r_{1}, \ldots, r_{l}$ слов, для которых $r_{1}=p, r_{l}=q$ и пара $\left(r_{i}, r_{i+1}\right)$ принадлежит классу хотя бы одного из семейств $v_{a}, v_{b}$. 
Теорема 2.5. Пусть существует гомоморфизм $\varphi$ фрагмента $R$ на эталон $A$, причем $M(A, \varphi)$ обладает следующими свойствами 1-3:

(1) каждое $v_{s} \in M(A, \varphi)$ является правильным частичным разбиением множества $X^{k}$, полнымм по $X$;

(2) если $a \neq b, a, b \in S$, то разбиение $\left[v_{a}+v_{b}\right]$ состочт из одного класса;

(3) семейство $M(A, \varphi)$ является простым.

Тогда $R$ является представлением эталона относительно класса $F_{n} u \iota$.

Рассмотрим определенно диагностируемый эталон порядка $k=1$. По фрагменту $R$ построим автомат $Q_{1}$ следующим образом. Отождествим во фрагменте $R$ состояния, которые порождают одно и то же вход-выходное слово длины 1. Получим в общем случае недетерминированный частичный автомат $R^{\prime}$. Затем проведем детерминизацию автомата $R^{\prime}$ точно так же, как при построении замыкания. Результат детерминизации обозначим через $Q_{1}$. Таким образом, автомат $Q_{1}$ является замыканием фрагмента $R$ по системе идентификаторов $(J, \sigma)$, где $J$ состоит из всех простых начальных идентификаторов длины 1 эталона, а $\sigma$ - диагональ, причем верификация этой системы не проводится.

Пусть существует гомоморфизм $\varphi$ фрагмента $Q_{1}$ на $A$. Рассмотрим семейство $M(A, \varphi)$. Условие 1 теоремы 2.5 равносильно тому, что каждое $v_{s}$ является разбиением множества $X$, условие 2 - тому, что для $a \neq b\left[v_{a}+v_{b}\right]=1$, где $1-$ единица решетки разбиений на множестве $X$, то есть разбиение, состоящее из одного класса $X$.

Имеет место следующий критерий.

Теорема 2.6. Фрагмент $R$ определенно диагностируемого порядка 1 эталона является его представлением относительно $F_{n}$ и ı тогда и только тогда, когда одновременно выполняются следуюшие условия.

(1) Фрагмент $Q_{1}$ не имеет висячих вершин, и $\varphi(Q)=A$ для всех гомоморфизмов $\varphi$ этого фрагмента в эталон.

(2) Семейство $M(A, \varphi)$ разбиений алфавита $X$, порожденное фрагментом $Q_{1}$, таково, что $\left[v_{a}+v_{b}\right]=1$, для всех $a \neq b$, где $a, b \in S$.

(3) Это семейство является простым.

В [6] показано, что при $n>2$ и $m>4$ условия 1-3 теоремы независимы.

\section{3. Представления определенно диагностируемых автоматов}

В настоящем разделе изучаются свойства правильных текстуальных представлений определенно диагностируемых порядка $k$ (ОД- $k$ ) автоматов. Выбор таких эталонов определяется следующими соображениями. ОД- $k$ автоматы обладают максимальным разнообразием внешнего поведения среди автоматов с одной и той же функцией переходов. В связи с этим кажется вероятным, что построение представлений таких эталонов менее трудоемко по сравнению с общим случаем. Кроме того, представление автомата с менее разнообразным поведением в большинстве случаев порождает представление ОД-1 автомата. Поэтому построение представления ОД-1 автомата можно рассматривать как промежуточный этап построения автомата с менее разнообразным поведением. 
В разделе сформулированы необходимые и достаточные условия (критерии), при которых фрагмент $R$ является представлением ОД-1 эталона относительно $F_{n}$ и различных $\tau$. Для случая $\tau=\iota$ описана структура экономных представлений. Такие представления позволяют строить экономные контрольные эксперименты и могут быть полезны при получении оценок длины контрольных экспериментов.

Найден критерий, при котором вход-выходное слово является контрольным экспериментом для ОД- $k$ эталона, $k \leqslant 11$, относительно $F_{n}$ и $\iota$.

Рассмотрим следующую задачу. Пусть $A$ - некоторый автомат с $n$ состояниями, и автомат $R$ гомоморфно отображается в $A$. Необходимо определить, является ли фрагмент $R$ представлением автомата $A$ относительно $n$-полного класса и $\tau=\iota$. В предыдущем разделе был предложен метод решения этой задачи при наличии некоторой информации о верифицированных в $R$ идентификаторах поведения состояний. Исходя из этой информации, по определенным правилам строилась последовательность автоматов, отражающая прирост информации в процессе анализа фрагмента $R$. Эта последовательность автоматов за конечное число шагов сходилась к единственному так называемому предельному автомату-замыканию $Q$. Вид замыкания $Q$ и определяет, является ли этот фрагмент представлением автомата $A$. Однако априорной информации об идентификаторах автомата $A$ может не быть. Тогда совокупность идентификаторов приходится определить по виду автомата $R$, что в общем случае является весьма сложной задачей. Уровень ее сложности определяется как свойствами автомата $A$, так и свойствами фрагмента $R$. С этой точки зрения ОД-1 автоматы являются идеальными объектами - по виду отображающихся в них фрагментов легко определить начальное множество верифицированных идентификаторов.

Пусть $R-$ фрагмент эталона, для которого выполняется равенство $\Phi_{A}^{1}=\Phi_{R}^{1}$. Из свойств класса $F_{n}$ вытекает, что каждое слово $(x, y) \in \Phi_{R}^{1}$ является простым начальным идентификатором состояний любого $B \in F_{n}$, для которого $R \leqslant B$. Поэтому такой автомат $B$ является ОД-1 автоматом. Множество $\Phi_{R}^{1}$ принимаем в качестве начального множества верифицированных в $R$ идентификаторов. Введем на множестве $T$ состояний фрагмента $R$ некоторые отношения. Пусть $\lambda_{R t}^{1}-$ множество всех вход-выходных слов длины 1 , порождаемых состоянием $t$ фрагмента $R$, а $\psi_{R t}^{1}-$ множество всех вход-выходных слов длины 1 , которые оканчиваются в этом состоянии. Полагаем $(s, t) \in \beta$, если $\lambda_{R t}^{1} \cap \lambda_{R s}^{1} \neq \varnothing$ или $\psi_{R t}^{1} \cap \psi_{R s}^{1} \neq \varnothing$, где $s, t \in T$. По определению $\beta$ рефлексивно и симметрично. Пусть $\alpha$ - транзитивное замыкание отношения $\beta$, то есть $\alpha=\bigcup_{i=0}^{\infty} \beta^{i}-$ наименьшая эквивалентность, для которой $\alpha \supseteq \beta$.

\section{Лемма 3.1. Если $R \leqslant A$, то $\alpha$ является конгруэничией на $T$.}

Конгруэнция $\alpha$ на состояниях фрагмента $R$ эталона $A$ однозначно определяет факторавтомат $R / \alpha$. Ясно, что $R \leqslant R / \alpha \leqslant A$. Если $R \leqslant A$ и $\Phi_{A}^{1}=\Phi_{R}^{1}$, то $R / \alpha$ является замыканием фрагмента по верифицированной системе $(J, \sigma)$, где $J=\Phi_{A}^{1}$ и $\sigma$ - диагональ. Автомату $R / \alpha$ поставим в соответствие неориентированный граф $G(R)$, полагая, что множеством его вершин является множество состояний этого автомата, то есть множество $T / \alpha$ классов конгруэнции $\alpha$, вершины $\alpha(a)$ и $\alpha(b)$ соединены ребром, если $\alpha(a) \neq \alpha(b)$, и функция выходов $\Lambda$ автомата $R / \alpha$ такова, что $\Lambda(\alpha(a), x), \Lambda(\alpha(b), x)$ одновременно определены для некоторого $x \in X$, и стало быть, $\Lambda(\alpha(a), x) \neq \Lambda(\alpha(b), x)$. Говорят, что граф $G(R)$ однозначно $n$-раскрашиваем, если все раскраски его вершин в $n$ цветов изоморфны [9].

Теорема 3.1. Автомат $R$ является представлением ОД-1 эталона относительно $F_{n} u \iota$ тогда и только тогда, когда одновременно выполняются следующие условия: 
(1) $R \leqslant A$;

(2) $\Phi_{A}^{1}=\Phi_{R}^{1}$;

(3) граф $G(R)$ однозначно раскрашиваем.

Эта теорема указывает, наряду с теоремой 2.6, конструктивный способ проверки того, является ли предъявленный фрагмент $R$ замыканием эталона относительно вышеуказанных $F$ и $\tau$. Проверка сводится к проверке однозначности раскраски, то есть к хорошо известной комбинаторной задаче. Конструктивные критерии “быть представлением” из теорем 2.6, 3.1 могут служить правилами завершения контрольных экспериментов различного вида: пассивного эксперимента при так называемом функциональном контроле $[32,41]$, когда экспериментатор только наблюдает входы и выходы автомата в процессе его штатного функционирования; активного эксперимента, когда на вход автомата поступает (псевдо-) случайная последовательность; активного тестового эксперимента, когда экспериментатор подает на автомат специально построенную входную последовательность $[32,41]$. Эти критерии полезны и при разработке алгоритмов построения таких последовательностей.

Напомним, что представление $R \in R_{\tau}\left(A, F_{n}, \iota\right)$ называется минимальным, если для любого представления $Q \in R_{\tau}\left(A, F_{n}, \iota\right)$ из неравенства $Q \leqslant R$ следует включение $R \subseteq Q$. Из определения вытекает, что минимальные представления не имеют гомоморфизмов в себя, кроме изоморфизмов, и не имеют изоморфных компонент связности. Другими словами, минимальные представления - это такие представления, в которых нельзя расщеплять и дублировать состояния или вычеркивать дуги, то есть минимальное представление это некоторый “неделимый” фрагмент функционирования эталона.

Правильной частью $O(R)$ автомата $R$ назовем автомат, полученный из $R$ удалением всех его изолированных дуг (но не петель).

Пусть $t \in T-$ некоторое состояние автомата $R$. Обозначим через $O(t)$ совокупность всех тех дуг автомата $R$, начала или концы которых совпадают с $t$. Каждую петлю $(t, x, y, t)$ из $O(t)$ заменим парой дуг $\left(t^{\prime}, x, y, t\right),\left(t, x, y, t^{\prime \prime}\right)$ и поместим их в $O(t)$. Все состояния в дугах из $O(t)$, отличные от $t$ (включая $\left.t^{\prime}, t^{\prime \prime}\right)$, переобозначим так, чтобы в разных дугах не встречалось одно и то же состояние. Каждое двухэлементное подмножество множества $O(t)$ образует частичный автомат, имеющий 3 состояния, у которого состоянию $t$ инцидентны две дуги, а остальным двум состояниям - по одной. Прямую сумму всех таких автоматов обозначим через $\Sigma O(t)$. Прямую сумму по всем $t \in T$ всех автоматов $\Sigma O(t)$ обозначим через $\Sigma R$. Ясно, что $\Sigma R \leqslant O(R) \subseteq R$.

Теорема 3.2. Автомат $R$ является представлением ОД-1 автомата А относительно $F_{n}$ и เ тогда и только тогда, когда $\Sigma R$ является представлением $A$.

Следствие 3.1. Если $R$ является минимальным представлением ОД-1 эталона относительно $F_{n}$ и ८, то $R=\Sigma R$.

Следствие 3.2. Для любого представления $R$ OД-1 эталона относительно $F_{n} u \iota c y$ ществует гомоморфно отображающееся на $R$ минимальное представление.

Теорема 2.6 дает критерий, при котором фрагмент $R$ является представлением ОД-1 эталона относительно $F_{n}$ и $\iota$ в терминах свойств разбиений $v_{s}$. Теорема 3.1 дает критерий в терминах однозначной раскраски вспомогательного графа, полученного на замыкании фрагмента по верифицированной системе $(J, \sigma)$, где $J=\Phi_{A}^{1}$ и $\sigma-$ диагональ множества $J$. Следующее утверждение показывает, что увеличение информации о совместимости верифицированных идентификаторов снимает необходимость исследования раскрасок 
вспомогательного графа. Пусть $R-$ некоторый фрагмент. Зафиксируем верифицированную систему $(J, \sigma)$, где $J$ состоит из всех верифицированных в $R$ простых начальных и конечных идентификаторов состояний длины 1 , а $\sigma$ равно сужению $\sigma_{R}$ на $J$. Замыкание фрагмента $R$ по $(J, \sigma)$ обозначим через $Q$.

Теорема 3.3. Фрагмент $R$ является представлением ОД-1 эталона относительно $F_{n} u$ ı тогда и только тогда, когда $Q=A$.

Эта теорема показывает, что верификация идентификаторов состояний равносильна проверке существования однозначной $n$-раскраски и равносильна проверке свойств разбиений $v_{s}$.

Рассмотрим условия, при которых фрагмент является представлением относительно различных $\tau$. Пусть $\tau=v_{1}$.

Следствие 3.3. Фрагмент $R$ является представлением ОД-1 относительно $F_{n}$ и $v_{1}$ тогда и только тогда, когда первые ядра эталона и замыкания $Q$ изоморфны и $\Phi_{A}^{1}=\Phi_{Q}^{1}$.

Рассмотрим случай $\tau=\varepsilon_{1}$.

Теорема 3.4. $R$ является представлением ОД-1 эталона относительно $F_{n}$ и $\varepsilon_{1}$ тогда $и$ только тогда, когда $\Lambda_{Q}^{1} \supseteq \Lambda_{A}^{1}$.

Для отношения неотличимости $\rho_{r i}$ автоматов экспериментами кратности $r$ и высоты $i$ на классе $F_{n}$ можно показать, что если $(A, B) \in \rho_{r i}$, то существует всего четыре возможности: $(A, B) \in \rho_{r 1},(A, B) \in v_{1},(A, B) \in \varepsilon_{1},(A, B) \in \varepsilon_{i}$. Очевидно, что $R$ является представлением ОД-1 эталона относительно $F_{n}$ и $\rho_{r 1}$ тогда и только тогда, когда $\Phi_{R}^{1}=\Phi_{A}^{1}$. Теоремы 3.1, 3.4 и следствие 3.3 дают соответствующие критерии для оставшихся случаев. Вместе они дают критерий, при котором $R$ является представлением ОД-1 эталона относительно $F_{n}$ и произвольного $\rho_{r i}$.

Заметим, что не только увеличение информации о верифицированной системе идентификаторов может снимать требование однозначной раскраски вспомогательного графа. Структура самого класса $F$, связанного с автоматом-эталоном определенным образом, может упрощать критериальные требования к представлениям. В качестве одного из таких примеров ниже приведен класс так называемых локально порожденных (эталоном) автоматов, изучавшихся в [42-44].

Рассмотрим класс А автоматов, порожденных локальными преобразованиями эталона $[6,42,43]$. Окрестностью $O_{s}$ состояния $s \in S_{A}$ назовем множество всех тех его состояний, которые достижимы из $s$ или из которых достижимо $s$ по входным словам длины, не большей 1. Пусть теперь автоматы из А получены из эталона заменой состояния $\delta_{A}(s, x)$ некоторым $t \in O_{s}$. Полученный класс является подклассом предыдущего. Ясно, что если в эталоне существует компонента связности, состоящая из одного состояния $s$, то $O_{s}=\{s\}$, и эта компонента присутствует в любом $B \in \mathbf{A}$. Правильной частью эталона $A$ назовем автомат $D$, полученный из $A$ удалением всех компонент связности, имеющих одно состояние.

Теорема 3.5. $R$ является представлением ОД-1 автомата-эталона относительно $A$ и $i$ тогда и только тогда, когда для его замыкания $Q$ справедливо включение $Q \supseteq D$.

В заключение раздела приведем еще один критерий для более широкого класса определенно диагностируемых автоматов, требующий, однако, усиления требований к системе верифицированных идентификаторов. Пусть $A-$ это ОД- $k$ автомат, и $w-$ фрагмент его 
поведения. Пусть теперь $(J, \sigma)$ - более мощная верифицированная в $w$ система (относительно $F_{n}$ и $i$ ), для которой $J$ состоит из всех простых начальных и конечных идентификаторов состояний, входящих в $J_{R}$, а $\sigma-$ сужение $\sigma_{R}$ на $J$, и $Q-$ замыкание фрагмента $R(w)$ по новой системе $(J, \sigma)$.

Теорема 3.6. Вход-выходное слово $w$ является контрольным экспериментом для ОД- $k$ эталона при $k \leqslant 11$ относительно $F_{n}$ u ı тогда и только тогда, когда замыкание $Q$ изоморфно эталону.

\section{4. Теоретико-графовые характеризации представлений автомата}

В данном разделе основное внимание уделяется задаче характеризации представлений автомата относительно подклассов $n$-полного класса автоматов, образующихся при наложении определенных ограничений на их структурно-алгебраические и поведенческие свойства. Показано, что теорема 3.1, дающая характеризацию представлений относительно $n$-полного класса, является представителем целого класса аналогичных утверждений, к получению которых можно подойти с некоторых общих позиций. При этом существенно используются понятия и методы теории бинарных отношений и графов, что позволяет в ряде случаев оценить относительную сложность задачи распознавания представлений в терминах теории $N P$-полноты [10].

Далее в этом разделе автомат $A=\left(S_{A}, X, Y, \delta_{A}, \lambda_{A}\right)$ есть детерминированный конечный автомат Мили, возможно, частичный. Все рассматриваемые автоматы имеют одинаковые входной алфавит $X$ и выходной алфавит $Y$. Для частичных автоматов снова предполагаются одинаковыми области определения функций переходов и выходов, которые обозначаем для автомата $A$ через $\operatorname{Dom} A$. Если $\varphi-$ гомоморфизм автомата $B$ в автомат $A$, то при $\varphi(B)=A$ автомат $B$ будем называть полным фрагментом автомата $A$.

При изучении множества $R(A, F, \tau)$ всех представлений автомата $A$ относительно класса $F$ с точностью $\tau$ полагаем, что $\tau-$ изоморфизм либо изоморфное включение, $F-$ подкласс класса $F_{n}$ всех автоматов с числом состояний $n$, причем эталон $A-$ приведенный автомат с $n$ состояниями.

Пусть автоматы $R, A$ связаны наличием гомоморфизма, $R \leqslant A, R=(S, X, Y, \delta, \lambda)$. Априори или в результате анализа автомата $R$ определяются два отношения на множестве его состояний, отношение совместимости и отношение несовместимости. Первое допускает отождествление состояний, а второе запрещает такое отождествление при гомоморфизме $A$ в любой автомат из класса $F$. В частности, такие отношения могут вводиться на основе верифицированных идентификаторов состояний, как это описано в предыдущем разделе.

\section{1. Отношения совместимости}

Отношение $\sigma$ на множестве состояний фрагмента $R$ назовем верифицированным отношением совместимости, если для любых его состояний $a, b$ и гомоморфизма $\varphi$ фрагмента $R$ в любой автомат $B \in F$ из того, что $(a, b) \in \sigma$, следует, что $\varphi(a)=\varphi(b)$. Такое отношение $\sigma$ может определяться как внутренними свойствами автомата $R$ и класса $F$ (например, верифицированными идентификаторами состояний), так и навязываться извне, за счет дополнительной информации, не проявляющейся явно в эксперименте с автоматами.

Пусть $\varphi$ - гомоморфизм $R$ в $B \in F$ и $\operatorname{ker} \varphi=\varphi \circ \varphi^{-1}-$ его ядро, являющееся конгруэнцией на множестве состояний автомата $R$. Этот гомоморфизм может быть не 
единственным. Пусть $\Phi-$ множество всех гомоморфизмов автомата $R$ в автомат $B$. Тогда

$$
\varepsilon_{\boldsymbol{B}}=\bigcap_{\varphi \in \Phi} \operatorname{ker} \varphi
$$

- наименьшая (по включению) конгруэнция среди всех конгруэнций, определяемых гомоморфизмами $R$ в $B$. Если $B$ пробегает все автоматы класса $F$, то пересечение всех конгруэнций $\varepsilon_{B}$ определяет конгруэнцию

$$
\varepsilon_{F}(R)=\bigcap_{B \in F} \varepsilon_{B}
$$

на множестве состояний автомата $R$.

Всякое подмножество $\sigma \subseteq \varepsilon_{F}(R)$ назовем верифицированным (относительно класса $F$ ) отношением совместимости на множестве состояний автомата $R$.

В общем случае $\sigma$ не является конгруэнцией, но может быть естественным образом расширено до нее, например, с помощью процесса, соответствующего детерминизации автомата $R$, определяемого отношением $\sigma$ совместимости его состояний, до конгруэнции $\sigma^{*}$, наименьшей среди всех конгруэнций, содержащих $\sigma$ [11]. По ней корректно (на основании утверждения, аналогичного лемме 3.1), определяется фактор-автомат автомата $R$, который назовем замыканием $R$ по $\sigma$ и обозначим $[R]_{\sigma}$.

Обозначим через $E_{F}(R)$ множество всех верифицированных конгруэнций, упорядоченное отношением включения.

Справедливо следующее утверждение [11].

Теорема 4.1. Множество $E_{F}(R)$ верифицированных конгруэничий есть решетка с операциями

$$
\inf \left(\varepsilon_{1}, \varepsilon_{2}\right)=\varepsilon_{1} \cap \varepsilon_{2}, \quad \sup \left(\varepsilon_{1}, \varepsilon_{2}\right)=\left(\varepsilon_{1} \cup \varepsilon_{2}\right)^{*},
$$

единицей $1=\varepsilon_{F}(R)$ и нулем $0=i_{s}, \varepsilon_{1}, \varepsilon_{2} \in E_{F}(R)$, где $i_{s}-$ диагональ множества состояний s автомата $R$, соответствующая пустому отношению совместимости.

Отношения совместимости $\sigma$ такие, что $\sigma^{*}=\varepsilon_{F}(R)$, несут максимальную информацию о возможности отождествления состояний, пустое отношение $\sigma, \sigma^{*}=i_{s}$ указывает на отсутствие какой-либо информации об отождествлении состояний.

Пусть $\sigma_{1}-$ верифицированное отношение совместимости, $R_{1}=[R]_{\sigma_{1}}$. Через $F_{R}$ обозначим множество тех автоматов $B \in F$, для которых $R \leqslant B$, то есть $F_{R}=\mathbf{F}(R) \cap F$, где $\mathbf{F}(R)$ - класс описываемых фрагментом $R$ автоматов из множества $\mathbf{A}(U)$.

Теорема 4.2. Если $\sigma$ - верифицированное относительно класса $F$ отношение на множестве состояний автомата $R$, то описываемый автоматом $R$ класс $F_{R}$ совпадает $c$ классом $F_{R_{1}}$, описываемым его фактор-автоматом $R_{1}=[R]_{\sigma}$. При этом всякий гомоморфизм $\varphi$ автомата $R$ в автомат $B \in F$ может быть представлен как $\varphi=\eta \circ \beta$, где $\eta$-естественный гомоморфизм $R$ в $R_{1}$, a $\beta$-гомоморфизм $R_{1}$ в $B$.

\section{2. Отношения несовместимости}

Отношение $\rho$ на множестве состояний фрагмента $R$ назовем верифицированным отношением несовместимости, если для любых его состояний $a, b$ и гомоморфизма $\varphi$ фрагмента 
$R$ в любой автомат $B \in F$ из того, что $(a, b) \in \rho$, следует, что $\varphi(a) \neq \varphi(b)$. Иными словами, если

$$
\mu_{B}=\bigcup_{\varphi \in \Phi} \operatorname{ker} \varphi,
$$

где объединение берется по всем гомоморфизмам $\varphi$ из $R$ в $B$, то $\rho-$ верифицированное отношение несовместимости тогда и только тогда, когда

$$
\rho \subseteq S \times S \backslash \bigcup_{B \in F} \mu_{B}=\rho_{F}(R)
$$

где объединение берется по всем $B \in F$, для которых существует гомоморфизм $R$ в $B$.

Пусть $\sigma-$ некоторое отношение совместимости на множестве состояний автомата $R$, а $\rho$ - отношение несовместимости на нем. Посредством отношения совместимости $\sigma$ отношение несовместимости может быть расширено (снова конструктивно) до некоторого максимального отношения $\rho^{*}$ несовместимости, согласованного с $\sigma$, которое назовем верифицированным замыканием $\rho$.

На множестве состояний $S / \varepsilon$ фактор-автомата $R_{1}=R / \varepsilon, \varepsilon=\sigma^{*}$, отношение $\rho^{*}$ индуцирует отношение верифицированной несовместимости $\rho_{1}$ по следующему правилу: $\left(\pi_{i}, \pi_{j}\right) \in \rho_{1}$, если в $\rho^{*}$ существует такая пара $(s, t)$, что $s \in \pi_{i}, t \in \pi_{j}$.

Множество верифицированных замыканий отношений несовместимости, согласованных с верифицированной конгруэнцией $\varepsilon$, упорядоченное отношением включения, обозначим через $P_{R}(F, \varepsilon)$. Оно имеет наибольшее верифицированное отношение несовместимости $\rho_{F}(R)$. Справедливо утверждение, аналогичное теореме 4.1.

Теорема 4.3. Для любой верифицированной конгруэнциии $\varepsilon \in E_{R}(F)$ множество $P_{R}(F, \varepsilon)$ есть решетка с операциями

$$
\inf \left\{\rho_{1}, \rho_{2}\right\}=\rho_{1} \cap \rho_{2}, \quad \sup \left\{\rho_{1}, \rho_{2}\right\}=\left(\rho_{1} \cup \rho_{2}\right)_{\varepsilon}^{*},
$$

$\rho_{1}, \rho_{2} \in P_{R}(F, \varepsilon)$, нулем, равным пустому отношению $\varnothing$, и единицей, равной $\rho_{F}(R)$.

\section{3. Ассоциированные графы}

Пару $P=(\sigma, \rho)$ верифицированных отношений совместимости $\sigma$ и несовместимости $\rho$ будем называть верифицированной парой для автомата $R$ (относительно класса $F$ ). Пусть $P=(\sigma, \rho)$ - верифицированная пара для $R$ относительно класса $F$. C $R$ и $P$ свяжем обыкновенный неориентированный граф $G(R, P)=\left(V_{R}, U_{R}\right)$, определенный следующим образом: множество его вершин $V_{R}$ есть множество $S / \varepsilon$ классов верифицированной конгруэнции $\varepsilon=\sigma^{*}$; если $v=[s]$ и $w=[t]-$ классы этой конгруэнции, представителями которой являются состояния $s$ и $t$ соответственно, и $(s, t) \in \rho_{\varepsilon}^{*}$, то $\{v, w\} \in U_{R}$.

На состояниях автомата $[R]$ отношение $\rho$ индуцирует верифицированное отношение несовместимости $\rho^{\prime}$ : если $[s]$ и $[t]-$ вышеуказанные классы, то есть состояния автомата $[R]_{\sigma}$, то $([s],[t]) \in \rho^{\prime}$ тогда и только тогда, когда $(s, t) \in \rho_{\varepsilon}^{*}$. Поэтому граф $G(R, P)$ можно понимать как задание индуцированного верифицированного отношения $\rho^{\prime}$ несовместимости на множестве состояний автомата $[R]_{\sigma}$, так как $\rho^{\prime}$ антирефлексивно и симметрично.

Граф $G(R, P)$ будем называть ассоциированным с автоматом $R$ посредством пары $P$ или, более коротко, ассоциированным с парой $(R, P)$. 
Пусть $G=(V, U)$ - некоторый обыкновенный неориентированный граф. Напомним, что правильной $n$-раскраской графа $G$ называется такое отображение $\varphi$ множества вершин графа в некоторое $n$-элементное множество $K$, при котором смежные вершины отображаются в разные элементы из $K$. Две раскраски $\varphi_{1}$ и $\varphi_{2}$ называются изоморфными, если $\operatorname{ker} \varphi_{1}=\operatorname{ker} \varphi_{2}$. Граф называется однозначно $n$-раскрашиваемым, если все его $n$-раскраски изоморфны [9].

Поставим в соответствие автомату $R=(S, X, Y, \delta, \lambda)$ семейство $X(R)$ подмножеств множества $X(R): X_{s} \in X(R), s \in S$, если $X_{s}=\{x \in X \mid(s, x) \in \operatorname{Dom} R\}$. Через $\Gamma(X(R))$ обозначим граф пересечений семейства $X(R)$, у которого множество вершин $V(X(R))$, и $\{s, t\} \in U(X(R))$, если $X_{s} \cap X_{t} \neq \varnothing$.

\section{4. Характеризация представлений и их сложность}

Далее задача идентификации автомата рассматривается при следующих ограничениях.

Все рассматриваемые классы состоят из всех приведенных автоматов в алфавите $U=X \times Y$. Автоматы, если не оговорено противное, неинициальные, связные, точность $\tau$ - изоморфизм, и если $A \in F-$ выделенный автомат-эталон, то он не вкладывается изоморфно ни в какой другой автомат $B \in F$.

Пусть заданы класс $F$, эталон $A \in F$ и его фрагмент $R$. Напомним, что фрагмент автомата $A$ есть его представление относительно $F$ (с точностью до изоморфизма), если $\mathbf{F}(R) \cap F=\{A\}$ (единственность понимается с точностью до изоморфизма), то есть идентификация осуществляется с точностью, равной изоморфизму.

Основной интерес представляют нетривиальные представления, не содержащие в явном виде информацию о полном функционировании анализируемого автомата.

Наиболее изученными среди нетривиальных представлений являются контрольные эксперименты, как простые, так и кратные. Такие представления обладают наиболее бедными отношениями совместимости и несовместимости, и по структуре графов переходов фрагментов их можно классифицировать как древовидные. Они образуют подкласс класса ациклических представлений, в которых возможно отождествление некоторых висячих вершин. В широком смысле циклические представления - это такие представления, которые в графе переходов имеют сильно связные подграфы, и класс тривиальных представлений является подмножеством этого класса.

Используя теорему 2.1, можно привести примеры, показывающие, что существование одного из видов представлений, например, нетривиальных циклических, не влечет существование, например, контрольных экспериментов (древовидных представлений).

Варьирование классами $F$ приводит к различным по сложности конкретным задачам описания соответствующих представлений. Само задание классов может осуществляться различными способами. В предыдущих разделах приведены варианты таких описаний с помощью различных дескрипторов автоматов.

Пусть $R \in R(A, F, \tau)$ - нетривиальное представление автомата $A$ и $\left|S_{A}\right|=n$. Верифицированная пара $P=(\sigma, \rho)$ для $R$ относительно $F$ определяет структуру раскрасок графа $G(R, P)$. Далее будем рассматривать только подклассы $F n$-полного класса и эталоны с $n$ состояниями. Назовем класс $F n$-плотным для эталона $A$, если для любого его фрагмента $R$ такого, что $\varphi(R) \neq A$ для некоторого гомоморфизма $\varphi$, следует, что $R$ есть фрагмент некоторого другого автомата $B$ из $F$ (более широкое понятие $n$-плотного класса рассмотрено в [45]). Потребуем, чтобы рассматриваемые классы были $n$-плотными для эталонов.

Покажем, что некоторые хорошо известные классы являются $n$-плотными для всех принадлежащих им автоматов. 
Теорема 4.4. Класс $F_{n}$ является n-плотным для любого принадлежащего ему автомата с п состояниями.

Пусть

$$
\Lambda_{A}^{i}=\left\{\lambda_{s}^{i} \mid s \in S_{A}\right\}
$$

и $\lambda-$ функция выходов автомата $A \in F_{n}$. Введем обозначение

$$
L_{n}^{i}(A)=\left\{B \in F_{n} \mid \Lambda_{B}^{i}=\Lambda_{A}^{i}\right\}
$$

Теорема 4.5. Класс $L_{n}^{1}(A)$ является $n$-плотным для $A$.

Класс $L_{n}^{1}(A)$ полностью определяется сохранением одношаговой функции выходов автомата $A$ при произвольном изменении функции переходов.

Другим предельным случаем можно считать сохранение функции переходов автомата $A$ при произвольном изменении функции выходов.

Обозначим через $D_{n}(A)$ множество всех автоматов из $F_{n}$, которые являются приведенными формами автоматов, полученных из $A$ всевозможными изменениями меток его дуг.

Теорема 4.6. Класс $D_{n}(A)$ является $n$-плотным для приведенного автомата $A$ с $n$ состояниями.

Пусть $A-$ ОД-1 автомат с $n$ состояниями и $F-n$-плотный для $A$ класс.

Теорема 4.7. $R \in R(A, F, \tau)$ тогда и только тогда, когда сущчествует такая верифицированная пара $P=(\sigma, \rho)$ для $R$ относительно $F$, что выполняются следуюшие условия:

(1) $R \leqslant A$;

(2) $\Phi^{1}(R)=\Phi^{1}(A)$;

(3) граф $G(R, P)$ однозначно п-раскрашиваем.

Приведенные достаточные и необходимые условия для распознавания представлений постулируют только существование верифицированной пары. Для ряда классов дается конструктивный способ построения таких верифицированных пар, позволяющий сводить задачу анализа фрагментов на свойство “быть представлением” к задаче раскраски некоторого графа.

Основным средством построения верифицированных пар являются идентификаторы состояний. Напомним, что фрагмент $R$ автомата $A$ с фиксированным состоянием $t$ называется идентификатором состояния $s$ автомата $A$, если для любого гомоморфизма $\varphi$ фрагмента $R$ в $A$ выполняется равенство $\varphi(t)=a$.

Как обычно, $\Phi_{A}$ обозначает множество всех вход-выходных слов, порождаемых автоматом $A$, а $\Phi_{A}^{k}-$ его подмножество из слов длины $k$. Если $R-$ некоторый, вообще говоря, частичный автомат, $s-$ его состояние, то $\lambda_{R s}^{k}$ есть множество всех вход-выходных слов длины $k$, порождаемых состоянием $s$;

$$
\Phi_{R}^{k}=\bigcup_{s \in S_{R}} \lambda_{R s}^{k} .
$$

Множество первых проекций $\lambda_{R s}^{1}$ и $\psi_{R s}^{1}$ обозначим через $X_{R s}^{+}$и $X_{R s}^{-}$соответственно (напомним, что $\psi_{R s}^{k}$ обозначает множество вход-выходных слов длины $k$, оканчивающихся 
в состоянии $s$ ). Если фрагмент $R$ ОД-1 автомата $A$ таков, что $\Phi_{R}^{1}=\Phi_{A}^{1}$, то множество $\Phi_{R}^{1}$ является множеством простых начальных идентификаторов состояний любого автомата $B \in F_{n}$, фрагментом которого является $R$, то есть при любом гомоморфизме $R$ в $B$ состояния автомата $R$, порождающие одну и ту же пару $(x, y) \in X \times Y$, отображаются в одно и то же состояние. Тогда на основании множества $\Phi_{R}^{1}$ как множества верифицированных идентификаторов (см. раздел 1) может быть определена верифицированная пара $P=(\sigma, \rho)$.

Пусть $A$ - ОД-1 автомат. Если $F=F_{n}, A \in F_{n}$ и $A$ есть ОД-1 автомат, то $\Phi_{A}^{1}$ является множеством верифицированных идентификаторов состояний автомата $A$ для любого полного фрагмента $R \leqslant A$. По этому множеству определяется пара верифицированных отношений $\sigma_{n}$ и $\rho_{n}$ на множестве состояний фрагмента $R:(a, b) \in \sigma$, если $\lambda_{R}(a, x)=\lambda_{R}(b, x)$ (то есть $\lambda_{R_{a}}^{\prime} \cap \lambda_{R_{b}}^{\prime} \neq \varnothing$ ) для некоторого $x \in X ;(a, b) \in \rho$, если $\lambda_{R}(a, x) \neq \lambda_{R}(b, x)$ для некоторого $x \in X$. Тогда $\sigma_{n}=\sigma^{*}, \rho_{n}=\rho_{\sigma_{n}}^{*}$ и $P_{n}=\left(\sigma_{n}, \rho_{n}\right)-$ верифицированная пара. По этой паре определяется ассоциированный граф $G\left(R, P_{n}\right)$, и из теорем 4.4 и 4.7 получаем, как следствие, теорему 3.1 , в которой $G(R)=G\left(R, P_{n}\right)$. Заметим, что в этом случае $G(R)=\Gamma(X(R))$.

Пусть теперь $A$ - групповой ОД-1 автомат, то есть $A$ такой, что при любом фиксированном $x \in X$ отображение $\delta_{A}(\cdot, x)$ есть перестановка на множестве $S_{A}, F=G_{n} \subseteq F_{n}-$ класс групповых автоматов и $R$ - полный фрагмент $A$, то есть при любом гомоморфизме $R$ в $A$ любая дуга из $A$ имеет прообраз в $R$.

Пусть на множестве $S_{R}$ заданы отношения $\sigma_{1}$ и $\rho_{1}$ следующим образом: $\sigma_{1}=\sigma$, $(a, b) \in \rho_{1}$, если для некоторого $x \in X$ либо $\lambda_{R}(a, x) \neq \lambda_{R}(b, x)$, либо существуют состояния $a^{\prime}$ и $b^{\prime}$ такие, что $\delta_{R}\left(a^{\prime}, x\right)=a, \delta_{R}\left(b^{\prime}, x\right)=b$ и $\lambda_{R}\left(a^{\prime}, x\right) \neq \lambda_{R}\left(b^{\prime}, x\right)$. Положим $\sigma_{g}=\sigma_{1}^{*}=\sigma_{n}, \rho_{g}=\rho_{1 \sigma_{g}}^{*}$. Тогда $P_{g}=\left(\sigma_{g}, \rho_{g}\right)$ - верифицированная пара.

Лемма 4.1. Класс $G_{n}$ является п-плотным для любого группового ОД-1 автомата при $|S| \geqslant 2,|X| \geqslant 2$.

Из леммы 4.1 и теоремы 4.7 вытекает следующее утверждение.

Теорема 4.8. Автомат $R$ есть представление группового ОД-1 автомата $A$ относительно класса $G_{n}$ тогда и только тогда, когда выполняются следующие условия:

(1) $R \leqslant A$;

(2) $\Phi_{R}^{1}=\Phi_{A}^{1}$;

(3) граф $G\left(R, P_{g}\right)$ однозначно п-раскрашиваем.

Из этой теоремы следует, что в отличие от случая, когда в качестве класса $F$ выбирается класс $F_{n}$ всех автоматов с $n$ состояниями, нетривиальные представления группового автомата относительно класса $G_{n}$ (точнее, их замыкания) допускают висячие вершины, и контрольные эксперименты, как частный случай представлений, могут содержать неподтвержденные переходы. На основании этой теоремы могут быть построены минимальные представления для этого случая.

Рассмотрим теперь представления автоматов без потери информации. Пусть $A-$ ОД-1 автомат и $A$ - автомат без потери информации порядка 1 (далее просто БПИ-автомат $[4,7])$, то есть $A$ таков, что для любого его состояния $s$ и различных $x_{1}, x_{2} \in X$ справедливо неравенство $\lambda_{A}\left(s, x_{1}\right) \neq \lambda_{A}\left(s, x_{2}\right)$. Пусть класс $I_{n} \subseteq F_{n}$ есть класс всех БПИ-автоматов. В этом случае для полного фрагмента $R \leqslant A$ определим отношения $\sigma_{\omega}, \rho_{\omega}$ следующим образом: $\sigma_{\omega}=\sigma_{n} ;(a, b) \in \rho_{\omega}$, если $(a, b) \in \rho_{n}$ или для некоторых $x_{1}, x_{2} \in X, x_{1} \neq x_{2}$, 
выполняется равенство $\lambda_{R}\left(a, x_{1}\right)=\lambda_{R}\left(b, x_{2}\right)$. Тогда $P_{\omega}=\left(\sigma_{\omega}, \rho_{\omega}\right)-$ верифицированная пара, и с этой парой ассоциирован граф $G\left(R, P_{\omega}\right)$. В этом случае для класса $I_{n}$ справедлив аналог леммы 4.1 и теоремы 4.8 с соответствующей заменой ассоциированных графов.

Заметим, что структура ассоциированного графа в этом случае является более регулярной по сравнению с предыдущими случаями.

Вернемся снова к представлениям локально порожденных автоматов, рассмотренных в предыдущем разделе.

Пусть $A-$ ОД-1 автомат и $F_{A}$ - множество всех автоматов, локально порожденных $A$. В классе $F_{n}$ можно выделить неизбыточное множество автоматов, из которых в совокупности порождается весь класс $F_{n}$. Такое множество назовем базисом локальной порождаемости класса $F_{n}$ и обозначим через $M_{n}$ (его структура описана в [44]).

Пусть $F=F_{A}$. Так как функция выходов $\lambda_{A}$ сохраняется при локальной порождаемости, в качестве множества верифицированных идентификаторов для ОД-1 автоматов можно выбрать множество

$$
\Lambda_{A}^{1}=\left\{\lambda_{A s}^{1} \mid s \in S_{A}\right\}
$$

где

$$
\lambda_{A s}^{1}=\left\{(x, y) \in X \times Y \mid \lambda_{A}(s, x)=y\right\} .
$$

Определим пару отношений $\sigma_{l}$ и $\rho_{l}$ на множестве состояний фрагмента $R \leqslant A:(a, b) \in \sigma_{l}$, если существует такое состояние $s \in S_{A}$, что $\lambda_{R a}^{1} \cup \lambda_{R b}^{1} \subseteq \lambda_{A s}^{1} ; \rho_{l}$ - отношение несовместимости состояний. Положим $\sigma_{l}=\sigma_{l}^{*}, \rho_{l}=\rho_{\sigma_{l}}^{*}$, и тогда $P_{l}=\left(\sigma_{l}, \rho_{l}\right)$ - верифицированная пара. Заметим, что здесь $\sigma_{l}^{*}=\sigma_{l}$.

В этом случае также справедлив аналог леммы 4.1.

Из определения пары $P_{l}$ в случае, когда $R$ - представление ОД-1 автомата, следует, что граф изоморфен полному графу $K_{n}$, где $n-$ число состояний автомата. Очевидно, что $K_{n}$ однозначно $n$-раскрашиваем. Тогда аналог теоремы 3.1 в рассматриваемом случае для связных автоматов приобретает следующий вид.

Теорема 4.9. Автомат $R$ есть представление ОД-1 автомата А относительно класса $F_{A}$ тогда и только тогда, когда выполняются следующие условия:

(1) $R \leqslant A$;

(2) $\Phi_{R}^{1}=\Phi_{A}^{1}$;

(3) граф $G\left(R, P_{l}\right)$ изоморфен графу $K_{n}$.

Если в качестве представлений будем рассматривать контрольные эксперименты, то для связных автоматов, обладающих такими экспериментами относительно класса $F_{A}$, из этой теоремы вытекает следующее утверждение.

Следствие 4.1. Слово $w \in(X \times Y)^{*}$ есть контрольный эксперимент ОД-1 автомата $A$ относительно класса $F_{A}$ тогда и только тогда, когда $w \in \Phi_{A}, u w=w_{1}(x, y)$, где $w_{1}-$ обход автомата A.

Полученный критерий для контрольных экспериментов позволяет дать еще одну характеризацию контрольных экспериментов ОД-1 автоматов относительно локально порожденного класса автоматов через уже упоминавшиеся доминирующие множества.

Пусть $F$ - некоторый класс автоматов, $A$ - выделенный в нем автомат-эталон. Доминирующим множеством класса $F$ называется такое его подмножество $F^{\prime}$, что контрольный эксперимент автомата $A$ относительно $F^{\prime}$ является таковым и относительно класса $F$. Наименьшее по мощности доминирующее множество называется минимальным. 
Пусть $F=F_{A}$ и $F_{1}(A) \subseteq F_{A}$ есть множество автоматов, непосредственно порожденных автоматом $A$, то есть множество таких автоматов, функции переходов которых отличаются от функций переходов автомата $A$ ровно для одной пары $(s, x) \in S \times X$. Каждой такой паре соответствует множество

$$
F_{1}(s, x)=\left\{B \in F_{1}(A) \mid \delta(s, x) \neq \delta_{B}(s, x)\right\} .
$$

Выберем из каждого класса $F_{1}(s, x)$ по представителю и образуем из них множество $R_{1}$.

Обозначим через $K(A)$ множество всех дуг $(s, x, y, t) \in A, \delta(s, x)=t, \lambda(s, x)=y$ таких, что частичный автомат $A^{\prime}$, из которого удалена хотя бы одна из этих дуг, не имеет обхода. Пусть $D_{A}$ - подавтомат автомата $A$, не содержащий одноэлементных компонент связности. Если $A \neq D_{A}$, то полагаем $K(A)=K\left(D_{A}\right)$. Удалим из множества $R_{1}$ все автоматы $B \in F_{1}(s, x)$, где $(s, x)$ соответствует дуге из $K(A)$, и получившееся множество обозначим через $R_{\min }$.

Теорема 4.10. Множество $R_{\min }$ есть минимальное доминирующее множество класса $F_{A}$ для ОД-1 автомата $A,\left|R_{\min }\right| \leqslant m n$, и существует автомат, для которого

$$
\left|R_{\min }\right|=m n-n+1
$$

В заключение рассмотрим характеризацию представлений ОД-1 автоматов относительно базиса $M_{n}$ класса $F_{n}$ по локальной порождаемости. При фиксированной функции выходов подмножество базиса, состоящее из всех автоматов с одной и той же функцией выходов $\lambda$, обозначим через $M_{\lambda}$ (автоматы полагаем определенными на одних и тех же множествах состояний $S$, входов $X$ и выходов $Y)$. В [44] показано, что при $m \leqslant 12 /(n-1)$, $m=|X|, n=|S|$ в автомате $A \in M_{\lambda}$ отсутствуют петли, параллельные дуги и циклы длины 2.

Пусть $A-$ ОД-1 автомат, $R$ - его фрагмент $A \in M_{n}$. Определим отношения $\sigma_{M}, \rho_{M}$ на множестве $S_{R}: \sigma_{M} \in \sigma_{n} ;(a, b) \in \rho_{2}$, если $(a, b) \in \rho$ либо расстояние между $a$ и $b$ в графе переходов $R$ без учета ориентации дуг равно 1 или 2 ; тогда $\rho_{M}=\rho_{2 \sigma_{M}}^{*}$, и $P_{M}=\left(\sigma_{M}, \rho_{M}\right)$ есть верифицированная пара, а $G\left(R, P_{M}\right)$ - ассоциированный граф.

В этом случае при $m \leqslant 12 /(n-1)$ справедливы аналоги леммы 4.1 и теоремы 4.8 .

В заключение раздела остановимся на задаче оценки сложности распознавания свойства “быть представлением”, при которых ключевую роль играют полученные теоремы характеризации представлений. На их основе проводится сведение указанной задачи к задаче анализа раскрасок некоторого графа и относительной оценки ее сложности в терминах $N P$-полноты. Используемые далее понятия теории $N P$-полноты понимаются в смысле [10].

Пусть для автомата $A$ определен некоторый класс $F_{A}$ автоматов. Задача распознавания представлений состоит в том, чтобы по заданному автомату $A$ и частичному автомату $R$ определить, будет ли $R$ принадлежать дополнению множества представлений автомата $A$ относительно класса $F_{A}$.

Исходной информацией для алгоритма, решающего задачу распознавания, будут пары $(R, A)$, которые можно закодировать в виде строк из нулей и единиц. Если автомат $A$ имеет $m$ входных сигналов, $n$ состояний и $l$ выходных сигналов, то для задания функций переходов и выходов достаточно строки длиной $m \log (n l)$, где $\log n-$ длина двоичной записи числа $n$. Если $R$ имеет $k$ состояний, то для его кодирования достаточно строки длиной $m k \log (k l+1)$ с учетом кодирования символа неопределенности. Таким образом, в конечном алфавите получим слово, описывающее исходную информацию для работы алгоритма распознавания. 
Результаты, касающиеся сложности задачи распознавания представлений для рассмотренных случаев [43, 46, 48, 49], могут быть суммированы в следующем утверждении.

Теорема 4.11. Задача распознавания представлений ОД-1 автомата в случаях п-полного класса, класса групповых автоматов, класса автоматов без потери информации, базиса локальной порождаемости является NP-полной. Для случая локальной порожденного класса она является полиномиальной.

Можно показать, что это утверждение справедливо и для частного случая представлений - контрольных экспериментов - во всех рассмотренных случаях. Кроме того, показано, что сужение класса БПИ-автоматов до класса автоматов существенно без потери информации [4] оставляет задачу распознавания автоматов в классе $N P$-полных задач.

Полученные теоремы характеризации контрольных экспериментов относительно различных классов автоматов позволяют получить нижние оценки их длин. Остановимся на случаях локально порожденного класса и $n$-полного класса.

Обозначим наименьшую возможную длину кратчайшего контрольного эксперимента относительно класса автоматов через $d_{\min }$, а длину наибольшего среди всех минимальных по всевозможным эталонам - через $d_{\max }$.

Теорема 4.12. Если $A-$ ОД-1 автомат и $F$-локально порожденный класс, то выполняются неравенства

$$
m n+1 \leqslant d_{\min } \leqslant d_{\max } \leqslant m n+\frac{1}{2}(m-1) n(n-1)+1,
$$

причем достижимы и нижняя и верхняя оценки.

Теорема 4.13. При $n \geqslant 2^{m-1}-1$ длина кратчайтего контрольного эксперимента $d_{\min }$ для любого ОД-1 автомата А относительно п-полного класса удовлетворяет неравенству

$$
d_{\min } \geqslant(2 m-1) n-2^{m-1}+2,
$$

причем оценка достижима при $m \geqslant 2, n \geqslant 2^{m-1}-1$.

Из теоремы 4.13 при $m=o(\log n)$ следует, что длина $d_{\min }$ асимптотически равна $(2 m-1) n$. В то же время относительно локально порожденного класса длина кратчайшего эксперимента равна $m n+1$ для любых $n$ и $m$. Таким образом, по нижним оценкам длины контрольного эксперимента имеется разрыв между локально порожденным и $n$-полным классами автоматов. Это связано с тем, что на структуре эксперимента для этого автомата в первую очередь проявляют себя особенности класса неисправностей, которые не “забиваются” свойствами структуры эталона. В то же время существуют автоматы, которые “затирают” особенностями своей структуры влияние класса неисправностей на структуру контрольного эксперимента. Так как на автоматах такого типа достигаются верхние оценки длины кратчайших экспериментов, эти оценки должны быть близки для локально порожденного и $n$-полного классов автоматов. Можно показать, что асимптотически верхние оценки длины кратчайших экспериментов относительно локально порожденного и $n$-полного классов автоматов совпадают. Это же справедливо и для случая базиса локальной порождаемости.

Таким образом, верхние оценки длин контрольных экспериментов ОД-1 автоматов относительно трех рассмотренных классов асимптотически равны. 


\section{5. Эксперименты в финитно-определенных классах}

В данном разделе изучаются свойства представлений - контрольных и распознающих экспериментов с автоматами относительно классов, названных финитно-определенными классами 1 и 2 рода. Финитно-определенными классами 1 рода названы классы автоматов, заданные недетерминированным автоматом-спецификацией, а финитно-определенными классами 2 рода - классы автоматов, наделенных специальными маркерами состояний (они были определены в разделе 1). Основным направлением исследований в этом случае является изучение связи топологических свойств этих классов с условиями существования и свойствами контрольных и распознающих экспериментов. Основным аппаратом исследования выступают введенные операторы аппроксимации.

В последнее время в качестве моделей дискретных систем выступают недетерминированные автоматы $[23,24,37,38,50,51]$. Это вызывает необходимость развития нового направления в теории экспериментов - экспериментов с недетерминированными автоматами.

В ряде работ (см., например, [50]) разрабатывались методы построения проверяющего теста для сети автоматов. При этом входы и (или) выходы исследуемой компоненты сети недоступны. Особенности проведения эксперимента с сетью автоматов следующие.

- Могут возникать неисправности, не обнаруживаемые на выходе сети, и такие неисправности исключаются.

- Не всякое слово может быть получено на входе тестируемой компоненты.

В [50] для исследования сетей автоматов предложен метод построения сетевого эквивалента компоненты сети - недетерминированного автомата, который полностью описывает поведение этой компоненты. Проверочный тест строится по этому эквиваленту, причем считается известной верхняя оценка числа состояний черного ящика.

В $[51,52]$ представлены два класса автоматов - класс работоспособных автоматов, реализуемых некоторым недетерминированным автоматом, и класс неработоспособных автоматов, представленный автоматами с числом состояний, не превосходящим известную верхнюю оценку. Так как неисправность автомата может значительно увеличить число состояний неисправного автомата, актуальна задача исследования недетерминированных автоматов при отсутствии верхней оценки числа состояний.

В [24] изучена проблема построения различающих экспериментов для классов, реализуемых двумя недетерминированными автоматами, причем первый описывает работоспособное, а второй - неработоспособное поведение исследуемого детерминированного автомата. Рассмотрены случаи как с известной, так и неизвестной верхней оценкой числа состояний.

Дальнейшее развитие эти исследования получили в [37, 38]. В них поставлена и решена проблема принадлежности исследуемого черного ящика, который является реализацией одного из двух недетерминированных автоматов, классу реализаций одного из них. В этих работах найдены конструктивные критерии существования таких алгоритмовэкспериментаторов и методы их построения.

Приведенные замечания показывают, что исследование финитно-определенных классов 1 рода достаточно важно и актуально. Для них получены критерии конечности (бесконечности) класса $F \in \mathbf{F}_{1}$ и его подклассов $\lim F$ и $] F$ [. Показана замкнутость множества $\mathbf{F}_{1}$ относительно оператора $\mathrm{lim}$. Найден ряд конструктивных критериев существования контрольных экспериментов относительно класса $G$ и автомата-эталона $A$, принадлежащих одному из классов $F, \lim F$ и $] F\left[\right.$ для некоторого $F \in \mathbf{F}_{1}$ для бэровской метрики, 
исследована финитная распознаваемость в метрике $\beta$ классов $F \in \mathbf{F}_{1}$ и его подклассов. Эти результаты получены в работах $[17,53,54,55]$.

Итак, исследуются подклассы класса $\mathbf{A}(U)$ всюду определенных инициальных, конечных, приведенных, инициально связных, детерминированных автоматов Мили. Приведем основные определения.

Пусть $G=\left(G, U, \delta, G_{0}\right)$ - всюду определенный слабо инициальный недетерминированный автомат (НДА), у которого $G$ - конечное множество состояний, $G_{0}$ - подмножество начальных состояний, $U=X \times Y-$ внешний алфавит, $\delta: G \times U \rightarrow 2^{G}$ - функция переходов. Обозначим через $g w=g(p, q)$ множество всех тех состояний, в которые НДА $G$ может переходить из состояния $g$ под действием входного слова $p$, порождая выходное слово $q$. Для $g \in G_{0}$ множество $L_{g}$ состоит из всех вход-выходных слов $w$ с непустым множеством $g w$. Множество $L_{g}^{k}$ есть сужение $L_{g}$ на слова длины, не большей $k$.

Слабо инициальный недетерминированный автомат $G$ называется наблюдаемым $[24,25]$, если для всех $u \in U, g \in G$ выполнено соотношение $|g u| \leqslant 1$, и инициально связным, если для всякого $g \in G$ существуют такие $g_{0} \in G_{0}, w \in L_{g_{0}}$, для которых $g \in g_{0}$. В дальнейшем, если не оговорено противное, под недетерминированным автоматом (НДА) будем понимать всюду определенный, слабо инициальный, наблюдаемый, инициально связный, недетерминированный автомат. Поведением НДА $G$ назовем

$$
L_{G}=\left\{L_{g} \mid g \in G_{0}\right\} .
$$

Инициальный автомат $A \in \mathbf{A}(U)$ есть реализация НДА $G$, если $L_{A} \subseteq L_{G}$. Класс всех реализаций НДА $G$ обозначим $R(G)$. При этом будем говорить, что $G$ определяет класс $R(G)$ или $G$ является его носителем. Класс автоматов $F \subseteq \mathbf{A}(U)$ назовем финитноопределенным классом 1-го рода и обозначим $F \in \mathbf{F}_{1}$, если существует НДА $G$, определяющий класс $F$. Напомним, что через $G_{g}$ обозначается подавтомат НДА $G$, получаемый объявлением состояния $g$ начальным. Ядром $\operatorname{Ker} G$ произвольного НДА $G$ назовем множество состояний $g$, для которых $G_{g}$ является инициальным детерминированным автоматом. Цикл в НДА $G$ есть пара $(g, w)$, где $g \in G$ и $w$ - вход-выходное слово, у которого $g=g w$.

Кроме этих классов, рассматриваются (возможно) бесконечные классы $\mathbf{A}(U, M)$ автоматов. Здесь $M \subseteq U$, и в каждом автомате из такого класса вход-выходной сигнал $(x, y) \in M$, называемый маркером, порождается не более чем одним состоянием, причем множество $M$ заранее известно экспериментатору. Маркеры присутствуют в ряде реальных автоматов в качестве дополнительных оповещающих сигналов или служебных режимов. Обозначим через $M_{x}$ множество всех маркеров $\left(x_{1}, y\right)$ с $x_{1}=x$. Класс автоматов $F$ назовем финитно-определенным классом 2-го рода и обозначим $F \in \mathbf{F}_{2}$, если существует множество маркеров $M$, для которого $\mathbf{A}(U, M)=F$. Класс $\mathbf{A}(U) \in \mathbf{F}_{2}$, так как $\mathbf{A}(U)=\mathbf{A}(U, \varnothing)$. Полагаем, что $\varnothing=\mathbf{A}\left(U, U \cup\left\{u_{1}\right\}\right)$, где $u_{1} \notin U$. Через $\mathbf{A}(U, n)$ обозначим подкласс $\mathbf{A}(U)$, состоящий из всех автоматов, число состояний которых не превосходит $n$.

\section{1. Алгебраические свойства множества финитно-определенных классов}

В подразделе исследуются структуры множеств $\mathbf{F}_{1}$ и $\mathbf{F}_{2}$. Введем над НДА операции прямой суммы и декартового произведения. Прямой суммой $G+H$ произвольных НДА $G$ и $H$ назовем НДА, получаемый расположением рядом $G$ и $H$ с предварительным переобозначением их состояний. Очевидно, что прямая сумма НДА $G$ и $H$ определяет класс автоматов $R(G) \cup R(H)$. 
Под декартовым произведением $G \times H$ НДА $G$ и $H$ будем понимать НДА $G \times H=$ $\left(G \times H, U, \delta_{G H}, G_{0}, H_{0}\right), \delta_{G H}: G \times U \rightarrow 2^{G} \times 2^{H}$, где $\delta_{G H}\left(\left(s_{1}, s_{2}\right), z\right)=\left(\delta_{G}\left(s_{1}, z\right), \delta_{H}\left(s_{2}, z\right)\right)$ при $\left.\delta_{G}\left(s_{1}, z\right) \neq \varnothing \neq \delta_{G}\left(s_{2}, z\right)\right)$ и $\delta_{G H}\left(\left(s_{1}, s_{2}\right), z\right)=\varnothing$ в противном случае.

Структура финитно-определенных классов 1-го рода дана в следующей теореме.

Теорема 5.1. Множество $\mathbf{F}_{1}$ является дистрибутивной решеткой относительно теоре-

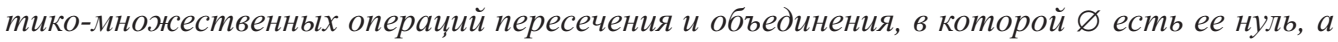
$\mathbf{A}(U)-$ единица.

Эта теорема расширяет аналогичный результат работ $[24,25]$ на случай слабо инициальных НДА.

Пусть $\beta-$ бэровская метрика на множестве $\mathbf{A}(U)$, введенная в [58] и определяемая следующим образом: $\beta(A, B)=0$, если $A=B$, и $\rho(A, B)=1 / k$, если $L_{A}^{k} \neq L_{B}^{k}$ и $L_{A}^{k-1}=L_{B}^{k-1}$. Пространство $(\mathbf{A}(U), \beta)$ будем называть бэровским пространством автоматов. Окрестностью с центром $A \in \mathbf{A}(U)$ и радиусом $r \in \mathbf{R}^{+}$назовем множество автоматов

$$
O_{r}(A)=\{B \mid B \in \mathbf{A}(U), \rho(A, B)<r\} .
$$

Автомат $A$ является предельным автоматом класса $F \subseteq \mathbf{A}(U)$, если для любого $r>0$ класс $O_{r}(A) \cap(F-\{A\})$ не пуст. Через $\lim F$ обозначим множество всех предельных автоматов класса $F$.

Пусть $r=|Y|$.

Следствие 5.1. (1) При $r>1$ множество $\mathbf{F}_{1}$ бесконечно и счетно.

(2) Всякий конечный класс $F$ принадлежит $\mathbf{F}_{1}$.

(3) Окрестности бэровского пространства автоматов и их конечное объединение принадлежат $\mathbf{F}_{1}$.

(4) Множество $\mathbf{F}_{1}$ не является булевой алгеброй относительно теоретико-множественных операчий пересечения, объединения и дополнения.

Часть 2 следствия 5.1 показывает коренное отличие введенного здесь понятия реализации от аналогичного понятия из $[23,24]$, для которого не всякий конечный класс реализуем инициальным недетерминированным автоматом.

Рассмотрим теперь структуру множества $\mathbf{F}_{2}$. Покажем, что это множество не замкнуто относительно операции объединения.

Предложение 5.1. Пусть $F_{1}, F_{2}-$ произвольные классы из $\mathbf{F}_{2}$. Справедливы следующие утверждения.

(1) Класс $F_{1} \cap F_{2} \in \mathbf{F}_{2}$.

(2) Объединение $F_{1} \cup F_{2}$ принадлежит множеству $\mathbf{F}_{2}$ точно тогда, когда $F_{1} \subseteq F_{2}$ или $F_{2} \subseteq F_{1}$.

Из утверждений 2 и 3 следует незамкнутость множества $\mathbf{F}_{2}$ относительно теоретикомножественного объединения. Введем операцию $\vee$ по правилу

$$
\mathbf{A}\left(U, M_{1}\right) \vee \mathbf{A}\left(U, M_{2}\right)=\mathbf{A}\left(U, M_{1} \cap M_{2}\right)
$$

для произвольных $M_{1}, M_{2}$ из $U$. 
Теорема 5.2. Существует изоморфизм булевой алгебры $\left\langle 2^{U}, \cap, \cup\right\rangle$ в булеву алгебру $\left\langle\mathbf{F}_{2}, \cap, \vee\right\rangle$.

Структура классов, являющихся финитно-определенными 1-го и 2-го рода одновременно, дана в следующем утверждении.

Теорема 5.3. Класс $F \in \mathbf{F}_{1} \cap \mathbf{F}_{2}$ точно тогда, когда $F$ nуст, совпадает с $\mathbf{A}(U)$ или является конечным классом из $\mathbf{F}_{2}$.

Из этой теоремы видно, что множества $\mathbf{F}_{1}$ и $\mathbf{F}_{2}$ описываются несводимыми друг к другу финитными средствами, что порождает достаточно выразительные множества классов автоматов.

Рассмотрим операторы алгебраического замыкания и аппроксимации, которые служат основным средством изучения контрольных и распознающих экспериментов в финитноопределенных классах 1-го и 2-го рода.

Оператор $\theta: 2^{\mathbf{A}(U)} \rightarrow 2^{\mathbf{A}(U)}$ называется оператором алгебраического замыкания [1], если выполнены следующие условия:

(1) для любого класса автоматов $F$ имеет место включение $F \subseteq \theta(F)$;

(2) для произвольного класса $F$ справедливо равенство $\theta(\theta(F))=\theta(F)$;

(3) для любых $F_{1}, F_{2} \subseteq \mathbf{A}(U)$ из $F_{1} \subseteq F_{2}$ вытекает включение $\theta\left(F_{1}\right) \subseteq \theta\left(F_{2}\right)$.

Алгебраическим замыканием класса $F$ относительно оператора $\theta$ назовем класс $\theta(F)$. Рассмотрим операторы $\theta_{i}, i=1,2$, ставящие в соответствие произвольному классу $F$ минимальный по включению класс $\theta_{i}(F) \in F_{i}, i=1,2$, который содержит $F$.

Предложение 5.2. Операторы $\theta_{i}, i=1,2$, являются операторами алгебрачческого замыкания.

Исследуем аппроксимацию произвольных классов $F$ классами вида $\theta_{1}\left(O_{\varepsilon}(A) \cap F\right)$, $A \in \mathbf{A}(U), \varepsilon \in \mathbf{R}^{+}$, для чего введем операторы аппроксимации $\theta_{F}: \mathbf{A}(U) \times \mathbf{R}^{+} \rightarrow \mathbf{F}_{1}$, удовлетворяющие следующим условиям:

(1) для любых $A \in \mathbf{A}(U), \varepsilon \in \mathbf{R}^{+}$справедливы включения

$$
O_{\varepsilon}(A) \cap F \subseteq \theta_{F}(A, \varepsilon) \subseteq O_{\varepsilon}(A)
$$

(2) из неравенств $0<\varepsilon_{1} \leqslant \varepsilon_{2}$ вытекает включение $\theta_{F}\left(A, \varepsilon_{1}\right) \subseteq \theta_{F}\left(A, \varepsilon_{2}\right)$;

(3) для любого $A \notin \lim F$ существует такое $\varepsilon>0$, что $\theta_{F}(A, \varepsilon) \subseteq F$.

В классе операторов аппроксимации фиксированного класса $F$ введем операции $\cap$ и $\cup$, полагая

$$
\begin{aligned}
& \left(\theta_{F}^{1} \cap \theta_{F}^{2}\right)(A, \varepsilon)=\theta_{F}^{1}(A, \varepsilon) \cap \theta_{F}^{2}(A, \varepsilon), \\
& \left(\theta_{F}^{1} \cup \theta_{F}^{2}\right)(A, \varepsilon)=\theta_{F}^{1}(A, \varepsilon) \cup \theta_{F}^{2}(A, \varepsilon) .
\end{aligned}
$$

Структура операторов аппроксимации дана в следующем утверждении.

Теорема 5.4. Множество операторов аппроксимации $\theta_{F}$ фиксированного класса является дистрибутивной решеткой относительно операций $\cap u \cup$, причем оператор $\theta_{F}(A, \varepsilon)=\theta_{1}\left(O_{\varepsilon}(A) \cap F\right)$ является ее нулем. 
Перейдем к рассмотрению условий существования контрольного эксперимента относительно $F \subseteq \mathbf{A}(U)$ и эталона $A \in \mathbf{A}(U)$. Напомним, что множество $W$ вход-выходных слов в алфавите $U$ называется контрольным экспериментом относительно $A$ и $F$, если $W \subseteq L_{A}$ и для всех $B \in F$ включение $W \subseteq L_{A}$ влечет изоморфизм автоматов $A$ и $B$.

Теорема 5.5. Пусть $\theta_{F}-$ произвольный оператор аппроксимации. В метрическом пространстве $\langle\mathbf{A}(U), \beta\rangle$ контрольный эксперимент относительно автомата-эталона $A$ $u$ класса $F$ существует точно тогда, когда существует $\varepsilon>0$, для которого $\theta_{F}(A, \varepsilon) \subseteq\{A\}$.

Теорема показывает, что проверку условия $O_{\varepsilon}(A) \cap F \subseteq\{A\}$ существования контрольного эксперимента для любого $F$ можно заменить проверкой справедливости включения $\theta_{F}(A, \varepsilon) \subseteq\{A\}$ для финитно-определенного класса $\theta_{F}(A, \varepsilon) 1$-го рода. Далее в данном разделе предложены алгоритмы такой проверки для бэровских метрик.

Из теоремы 5.5 нетрудно получить следующее утверждение.

Предложение 5.3. Дан оператор $\theta_{F}$ аппроксимациии и метрика $\rho$. Справедливы следующие утверждения.

(1) Контрольный эксперимент относительно автомата-эталона $A \in F$ класса $F$ существует тогда и только тогда, когда существует $\varepsilon>0$, для которого $\theta_{F}(A, \varepsilon)=\{A\}$.

(2) Автомат $A \in \lim F$ тогда и только тогда, когда для любого $\varepsilon>0$ справедливо неравенство $\left|\theta_{F}(A, \varepsilon)\right|>1$.

В теории автоматов, кроме условий существования контрольных экспериментов, требуется знать алгоритм их проведения. Для метрики $\beta$ справедлив следующий результат.

Теорема 5.6. Дан класс $F \in \mathbf{F}_{1} \cup \mathbf{F}_{2}$. Алгоритм построения носителя класса $\theta_{F}(A, \varepsilon)$ существует для любых $A \in \mathbf{A}(U), \varepsilon \in \mathbf{R}^{+}$, и бэровской метрики $\beta$.

Полученные утверждения позволяют единообразно исследовать контрольные эксперименты в финитно-определенных классах и получать алгоритмы их проведения. На базе этих теорем найдены конструктивные критерии и алгоритмы построения таких экспериментов.

\section{2. Эксперименты в финитно-определенных классах 1 рода}

В данном подразделе исследуются классы $F \in \mathbf{F}_{1}, \lim F$ и $] F[$ в бэровском пространстве $(\mathbf{A}(U), \beta)$ автоматов. Выбор метрики $\beta$ объясняется наличием для нее алгоритмов построения операторов аппроксимации и важностью ее для практических целей. Кроме того, как было показано, топологические свойства классов автоматов тесно связаны с условиями существования контрольных экспериментов. Справедливо следующее простое утверждение.

Лемма 5.1. Пусть НДА $G$ определяет $F$. Тогда класс $F$ рекурсивен.

Очевидно, что не всякий бесконечный класс автоматов содержит все собственные предельные автоматы, но для классов $F$ из $\mathbf{F}_{1}$ и $[F]=F \cup \lim F$ справедливо следующее утверждение.

Предложение 5.4. Пусть НДА $G$ определяет $F$. Класс $[F]$ определяется этим же автоматом $G$, то есть замкнут по предельным автоматам. 
Рассмотрим конструктивный критерий конечности классов из $\mathbf{F}_{1}$ в терминах свойств ядра его носителя.

Теорема 5.7. Пусть НДА $G$ определяет класс $F$. Класс F бесконечен точно тогда, когда в $G$ существует хотя бы один ичикл вне $\operatorname{Ker} G$.

Данное утверждение расширяет аналогичный результат из [25] на случай слабо инициальных недетерминированных автоматов.

По предложению 5.3, наличие контрольного эксперимента относительно автоматаэталона $A$ и класса $F$ тесно связано с условием принадлежности $A$ классу $\lim F$. Конструктивный критерий проверки условия $A \in \lim F$ для $F \in \mathbf{F}_{1}$ дает следующая теорема.

Теорема 5.8. Пусть НДА $G$ определяет класс $F$. Автомат A с начальным состоянием является предельным автоматом класса $F$ точно тогда, когда сущеествует такое начальное состояние $g_{0} \in G_{0}$ и вход-выходные слова $w, w^{\prime}$, для которых $\left(g_{0} w^{\prime}, w\right)-$ ичкл вне ядра $G$ и $a_{0} w^{\prime} w=a_{0} w^{\prime}, w^{\prime} w \in L_{A}$.

Данный результат показывает, что критерий существования контрольных экспериментов для классов из $\mathbf{F}_{1}$ является конструктивным. На этой основе получены алгоритмы проведения контрольных экспериментов. Существует также алгоритм проверки принадлежности $A$ классу $\lim F$ для любого $F \in \mathbf{F}_{1}$. Таким образом, класс $\lim F$, а значит, и его открывание - класс $] F[=F-\lim F$ являются рекурсивными. Более того, для класса $F$ с носителем $G$ класс $\lim F$ определяется некоторым НДА $H$, алгоритм построения которого также получен.

Теорема 5.9. Пусть НДА $G$ определяет $F$. Тогда $\lim F \in \mathbf{F}_{1}$.

Открывания классов из $\mathbf{F}_{1}$, как будет показано далее, играют центральную роль в изучении контрольных и распознающих экспериментов. Критерий принадлежности $] F\left[\in \mathbf{F}_{1}\right.$ для $F \in \mathbf{F}_{1}$ дает следующее утверждение.

Предложение 5.5. Пусть НДА $G$ определяет $F$. Класс $] F\left[\right.$ принадлежит $\mathbf{F}_{1}$ тогда $u$ только тогда, когда $F$ конечен.

Приведенные результаты показывают, что ряд мощностных и топологических свойств классов из $\mathbf{F}_{1}$, в отличие от свойств произвольных подклассов класса $\mathbf{A}(U)$, проверяется конструктивно.

Остановимся теперь на некоторых свойствах распознающих экспериментов в классах $G \in \mathbf{F}_{1}, G=\lim F$ и $\left.G=\right] F$ [ для некоторого $F \in \mathbf{F}_{1}$. Напомним понятие распознающего эксперимента для класса $F$ (в бэровской метрике). Алгоритмом распознавания автоматов из $F$ называется алгоритм, который для произвольного автомата $A \in F$ выполняет по шагам следующие действия: на каждом $i$-м шаге, $i \geqslant 1$, на автомат $A$ подаются слова из конечного множества $P_{i}$, регистрируются реакции автомата $A$ на эти слова, то есть регистрируется сужение $L_{A} / P_{i}$ поведения $L_{A}$ на множество $P_{i}$, и на основе этого сужения (и априорной информации о классе $F$ ) и таких же сужений, полученных на предыдущих шагах эксперимента, делается одно из двух возможных заключений:

(a) алгоритм завершен, и выдается таблица автомата $A$ (номер автомата $A$ в выбранной эффективной нумерации автоматов);

(б) алгоритм не завершен, и порождается множество $P_{i+1}$ входных слов. 
При этом, для каждого $A \in F$ алгоритм завершается через конечное число шагов. Класс $F$ назовем финитно-распознаваемым, если для него существует некоторый алгоритм распознавания. Пустой класс считается финитно-распознаваемым.

В [37] показано, что равносильны следующие утверждения.

(1) Класс $F$ является финитно-распознаваемым.

(2) Этот класс является подклассом рекурсивно-перечислимого финитно-распознаваемого класса.

(3) Для любого $A \in F$ существует эффективный контрольный эксперимент относительно $A, F$ и $\tau=\iota$.

Характеризацию множества всех финитно-распознаваемых классов из $\mathbf{F}_{1}$ дает следующее утверждение.

Предложение 5.6. Пусть $F \in \mathbf{F}_{1}$. Класс $F$ финитно распознаваем точно тогда, когда он конечен.

Исследуем теперь распознаваемость дополнений классов из $\mathbf{F}_{1}$.

Предложение 5.7. Если класс $F$ принадлежит $\mathbf{F}_{1}$ и его дополнение не пусты, то класс $\bar{F}$ не является финитно-распознаваемым.

Рассмотрим различные подклассы классов из множества $\mathbf{F}_{1}$. Следующая теорема описывает структуру всех финитно-распознаваемых подклассов конечного объединения непересекающихся классов из $\mathbf{F}_{1}$.

Теорема 5.10. Пусть $F_{1}, \ldots, F_{n}-$ попарно непересекающиеся классы из $\mathbf{F}_{1}$. Равносильны следуюшие утверждения:

(1) Класс $Н$ является финитно-распознаваемьлм подклассом объединения классов $\bigcup_{1 \leqslant k \leqslant n} F_{k}$;

(2) $H=\bigcup_{1 \leqslant k \leqslant n} H_{k}$, где $H_{k}$ являются финитно-распознаваемыми подклассами $F_{k}$ при $1 \leqslant k \leqslant n$.

В [17] показано, что в бэровской метрике открывание ] $F$ [ произвольного класса $F \in \mathbf{F}_{1} \cup \mathbf{F}_{2}$ финитно-распознаваемо.

Там же показано, что в общем случае не существует максимального по включению финитно-распознаваемого класса. Оказывается, что в множестве всех финитнораспознаваемых подклассов $F$ также в общем случае нет максимального.

Предложение 5.8. Пусть даны класс $F \in \mathbf{F}_{1}$ и рекурсивно перечислимый финитнораспознаваемый класс $H \subseteq F$, причем класс $] F[-H \cap] F[$ не пуст. Тогда существует такой рекурсивно перечислимьй финитно-распознаваемый класс $H_{1}$, что $F \supset H_{1} \supset H$.

\section{3. Эксперименты в финитно-определенных классах 2-го рода}

Далее исследуются свойства классов $\mathbf{A}(U, M), M \subseteq U$, автоматов, в каждом из которых вход-выходной сигнал $(x, y) \in M$, называемый маркером, порождается не более, чем одним состоянием, и $M$ заранее известно экспериментатору, то есть маркеры можно понимать как верифицированные идентификаторы состояний. При этом под автоматами понимаются всюду определенные, инициально связные автоматы из $\mathbf{A}(U, M)$.

2 Дискретная математика, т.21 №2 
Для классов автоматов $F \in \mathbf{F}_{2}$ (то есть таких, что для некоторого множества $M$ маркеров $\mathbf{A}(U, M)=F)$ найдены конструктивные критерии их конечности или бесконечности в терминах свойств множества маркеров. Рассматриваются кратные контрольные эксперименты, и найдены условия существования или несуществования таких экспериментов для автомата относительно класса $\mathbf{A}(U, M)$, дополнения его подкласса $\mathbf{B}(U, M)$ всех автоматов, в каждом цикле которых существует состояние, порождающее некоторый маркер, его подкласса $\mathbf{C}(U, M)$ всех автоматов, не порождающих ни одного маркера и т. п. Найден критерий (в терминах структуры графа автомата-эталона) существования простого контрольного эксперимента относительно класса $\mathbf{A}(U, M)$.

Рассмотрены условия существования или несуществования распознающих экспериментов. Показано, что класс $\mathbf{B}(U, M)$ является максимальным подклассом класса $\mathbf{A}(U, M)$, для которого такой эксперимент существует. Обнаружено, что для класса $\mathbf{C}(U, M)$ и дополнения класса $\mathbf{A}(U, M)$ распознающих экспериментов нет. Полученные результаты изложены в работах $[17,56,57]$.

Пусть дан класс $\mathbf{A}(U, M) \in \mathbf{F}_{2}$. Нетрудно привести примеры бесконечного, конечного и состоящего из одного элемента класса $\mathbf{A}(U, M)$. Просмотром таблицы автомата $A \in \mathbf{A}(U, M)$ легко обнаружить, входит этот автомат в класс $\mathbf{A}(U, M)$ или нет, поэтому $\mathbf{A}(U, M)$ рекурсивен. Автомат $A$ из этого множества назовем насыщенным, если для всякого маркера $m \in M$ в автомате существует отмеченное $m$ состояние. Пусть $r=|Y|$.

Лемма 5.2. Для всех $M$ класс $\mathbf{A}(U, M)$ содержит насыщенный автомат с не более чем $r$ состояниями.

Обозначим через $\mathbf{A}_{H}(U, M)$ подкласс всех насыщенных автоматов из $\mathbf{A}(U, M)$. Пустой класс будем считать конечным. Справедлива следующая теорема.

Теорема 5.11. Равносильны следуюшие утверждения.

(1) Все автоматы в $\mathbf{A}(U, M)$ насыщены.

(2) $\mathbf{A}(U, M)=\mathbf{A}(U)$.

(3) $\mathbf{A}(U, r) \subseteq \mathbf{A}(U, M)$.

(4) $r=1$ или М пусто.

(5) $\overline{\mathbf{A}}(U, M)$ - конечное множество.

Из утверждений 2 и 5 этой теоремы следует, что класс $\bar{A}(U, M)$ или пуст (если выполняется утверждение 4 теоремы) или бесконечен (в противном случае).

Напомним, что автомат можно отождествить с его графом, вершинами которого являются состояния, а дугами - тройки $(a, u, b)$, где $u \in U$ и $a u=b$. Циклом называется последовательность дуг $\left(a_{1}, u_{1}, a_{2}\right),\left(a_{2}, u_{2}, a_{3}\right), \ldots,\left(a_{k}, u_{k}, a_{1}\right)$. При этом говорим, что эти дуги и вершины $a_{1}, \ldots, a_{k}$ принадлежат этому циклу. Введем подкласс $\mathbf{B}(U, M)$ всех автоматов из $\mathbf{A}(U, M)$, у которых в каждом цикле графа имеется отмеченное состояние. Можно показать, что $\mathbf{B}(U, M)$ не пуст тогда и только тогда, когда $M$ не пусто. Проверка соотношения $A \in \mathbf{B}(U, M)$ легко осуществляется по графу автомата $A$, поэтому подкласс $\mathbf{B}(U, M)$ рекурсивен.

Рассмотрим условия конечности классов $\mathbf{A}(U, M)$ и $\mathbf{B}(U, M)$. Входное слово $p$ называется диагностическим для автомата $A$, если $a \neq b$ влечет $\lambda(a, p) \neq \lambda(b, p)$ для всех $a, b \in A$. Справедлива следующая теорема. 
Теорема 5.12. Равносильны следующие утверждения.

(1) Класс $\mathbf{A}(U, M)$ конечен.

(2) Подкласс насыщенных автоматов конечен.

(3) $\mathbf{A}(U, M) \subseteq \mathbf{A}(U, r)$.

(4) $r=1$ или $\left|M_{x}\right|=r$ для некоторого $x \in X$.

(5) Сущуествует $x$, являющийся диагностическим словом для всех $A \in \mathbf{A}(U, M)$.

(6) Каждый насыщенный автомат имеет ровно $r$ состояний.

Обозначим разность $\mathbf{A}(U, M)-\mathbf{B}(U, M)$ через $\mathbf{D}(U, M)$.

Теорема 5.13. Пусть $М$ не пусто. Равносильнь следующие утверждения.

(1) Класс $\mathbf{A}(U, M)$ конечен.

(2) Класс $\mathbf{B}(U, M)$ конечен и не пуст.

(3) Класс $\mathbf{D}(U, M)$ конечен.

(4) $\mathbf{A}(U, M)=\mathbf{B}(U, M)$.

Приведенные теоремы дают конструктивные критерий конечности классов $\mathbf{A}(U, M)$ и $\mathbf{B}(U, M)$ и характеризуют ее с различных сторон. Из этих теорем следует, что при непустом $M$ разность $\mathbf{D}(U, M)$, аналогично разности $\overline{\mathbf{A}}(U, M)$, либо пуста, либо бесконечна. При пустом $M$ эта разность или бесконечна, или состоит из одного автомата.

Следствие 5.2. Равносильны следующие утверждения.

(1) $\mathbf{A}(U, r)=\mathbf{A}(U, M)$.

(2) $\mathbf{A}(U, r)=\mathbf{A}(U)$.

(3) $r=1$.

Пусть $Z \subseteq X$. Обозначим $\mathbf{A}(U, r, Z)$ подкласс всех тех автоматов из класса $\mathbf{A}(U, r)$ автоматов с не более чем $r$ состояниями, для которых каждое $x \in Z$ является диагностическим словом.

Теорема 5.14. Пусть $Z$ не пусто. $M=Z \times Y$ тогда и только тогда, когда

$$
\mathbf{A}(U, M)=\mathbf{A}(U, r, Z)
$$

Следствие 5.3. $M=U$ тогда и только тогда, когда $\mathbf{A}(U, M)$ совпадает с классом определенно-диагностируемых порядка 1 автоматов.

Рассмотрим подкласс $\mathbf{C}(U, M)$ класса $\mathbf{D}(U, M)$ всех тех автоматов, каждый из которых не имеет отмеченных состояний и его дополнение

$$
\mathbf{H}(U, M)=\mathbf{D}(U, M)-\mathbf{C}(U, M) .
$$

Следующие теоремы дают критерии пустоты этих классов. 
Теорема 5.15. Равносильны следующие утверждения.

(1) Kласс $\mathbf{C}(U, M)$ nycm.

(2) Kласс $\mathbf{B}(U, M)$ nycm.

(3) $\left|M_{x}\right|=r$ для некоторого $x \in X$.

Теорема 5.16. Равносильны следующие утверждения.

(1) Kracc $\mathbf{H}(U, M)$ nycm.

(2) Этот класс конечен.

(3) Пустьл $M$ или $\mathbf{C}(U, M)$.

Эта теорема показывает, что класс $\mathbf{H}(U, M)$ либо пуст, либо бесконечен. В отличие от него класс $\mathbf{C}(U, M)$ может быть бесконечным, пустым и содержать при $r=1$ и пустом $M$ ровно один автомат. Рассмотрим критерий бесконечности этого класса.

Теорема 5.17. Равносильны следующие утверждения.

(1) Класс $\mathbf{C}(U, M)$ бесконечен.

(2) Этот класс содержит более одного автомата.

(3) $r>1,\left|M_{x}\right|<r$ для всех х $и\left|M_{x}\right|<r-1$ для некоторого $x$.

Следствие 5.4. Равносильны следующие утверждения.

(1) Класс $\mathbf{C}(U, M)$ состоит из одного автомата.

(2) $\left|M_{x}\right|=r-1$ для всех $x \in X$.

(3) Класс $\mathbf{C}(U, M)$ не пуст и каждый автомат из этого класса имеет ровно одно состояние.

В заключение отметим, что проверка конечности (бесконечности) и одноэлементности рассмотренных классов проводится по $M$ и $U$ конструктивно за полиномиальное время.

С целью получения условий существования контрольных экспериментов рассмотрим свойства различимости автоматов. Будем говорить, что автоматы $A$ и $B k$-неотличимы, и писать $(A, B) \in \varepsilon_{k}$, если $L_{A}^{k}=L_{B}^{k}$. Как и ранее, через $O_{1 / k}(A)$ обозначим класс всех автоматов из $\mathbf{A}(U), k$-неотличимых от $A$.

Лемма 5.3. Пусть $M$ не пусто. Для любого автомата $A \in \mathbf{A}(U)$ и натурального $k$ множество $O_{1 / k}(A) \cap \overline{\mathbf{A}}(U, M)$ бесконечно.

Следствие 5.5. При непустом $M$ не существует контрольного эксперимента для $A \in \mathbf{A}(U) u \overline{\mathbf{A}}(U, M)$.

Лемма 5.4. $O_{1 / k}(A) \subseteq \overline{\mathbf{A}}(U, M)$ для любого эталона $A \in \overline{\mathbf{A}}(U, M) u k \geqslant 2 n-2$.

Непосредственно из этой леммы вытекает следующее утверждение.

Следствие 5.6. Для каждого $A \in \overline{\mathbf{A}}(U, M)$ и $\mathbf{A}(U, M)$ сущчествует контрольный эксперимент кратности, не большей 4, и длинь,, не больщей $2 n-2$. 
Пусть даны класс $\mathbf{A}(U, M)$ и натуральное $k$. Справедлив следующий критерий существования контрольного эксперимента для $A \in \mathbf{A}(U, M)$.

Теорема 5.18. Равносильны следующие утверждения.

(1) Существует контрольный эксперимент для $A \in \mathbf{A}(U, M) u \mathbf{A}(U, M)$.

(2) Сущеествует контрольньй эксперимент для $A$ и $\mathbf{A}(U, M(A))$.

(3) $A \notin \mathbf{D}(U, M)$.

(4) $L_{A}^{k}$ является контрольным экспериментом для $A$ и $\mathbf{A}(U, M(A))$ nри всех $k \geqslant 2 n$.

Заметим, что класс $\mathbf{A}(U, M(A))$ может быть бесконечным при конечном классе $\mathbf{A}(U, M)$.

Из теоремы 5.18 вытекает, что для $A \in \mathbf{D}(U, M)$ и $\mathbf{A}(U, M)$ контрольных элементов не существует. Рассмотрим условия существования контрольных экспериментов для таких эталонов и подклассов этого класса автоматов.

Пусть $W$ - некоторое конечное подмножество множества $L_{A}$. Через $D(W)$ обозначим сужение информационного дерева автомата на это подмножество.

Теорема 5.19. $W$ является контрольным экспериментом для $A \in \mathbf{A}(U)-\mathbf{C}(U, M) u$ $\mathrm{C}(U, M)$ тогда и только тогда, когда дерево $D(W)$ содержит хоть одно отмеченное состояние.

Из этой теоремы вытекает, что для $A \in \mathbf{H}(U, M)$ и $F \subseteq \mathbf{C}(U, M)$ контрольный эксперимент существует. Более того, как будет отмечено далее, в этом случае существует и простой (однократный) эксперимент.

Теорема 5.20. Для $A \in \mathbf{C}(U, M)$ и непустого $\mathbf{H}(U, M)$ не существует контрольного эксперимента.

Теорема 5.21. Пусть $\mathbf{C}(U, M)$ содержит больше одного автомата. Для $\mathbf{C}(U, M) u$ $A \in \mathbf{C}(U, M)$ не существует контрольного эксперимента.

Из теорем 5.12 и 5.13 вытекает, что при $r>1$ класс $\mathbf{D}(U, M)$ или пуст, или бесконечен. Теорема 5.15 утверждает, что если $\mathbf{D}(U, M)$ не пуст, то не пуст и $\mathbf{C}(U, M)$. Теоремы 5.20, 5.21 показывают, что для $A \in \mathbf{C}(U, M)$ и $\mathbf{D}(U, M)$ контрольных экспериментов не существует. По теореме 5.18 , длина кратчайших контрольных экспериментов для $A \in \mathbf{B}(U, M)$ и $\mathbf{A}(U, M)$ не превосходит $2 n$. Можно показать, что для всех $n$ эта оценка достижима.

Рассмотрим случай, когда $M=U$. При этом $\mathbf{A}(U, M)=\mathbf{B}(U, M) \subseteq \mathbf{A}(U, r)$, и все автоматы из $\mathbf{A}(U, M)$ являются определенно диагностируемыми порядка 1 . Рассмотрим условия, при которых конечное подмножество $W \subseteq L_{A}$ является контрольным экспериментом для $A$ и $\mathbf{A}(U, M)$. По замыканию $B=[D(W)]_{U}$ и гомоморфизму $\psi$ замыкания $B$ в эталон построим вспомогательный неориентированный граф $G(W)$, у которого множество вершин $B$ и вершины $b_{1}, b_{2}$ графа соединены ребром, если $\psi\left(b_{1}\right) \neq \psi\left(b_{2}\right), \lambda_{B}\left(b_{1}, x\right), \lambda_{B}\left(b_{2}, x\right)$ одновременно определены для некоторого $x$ и, стало быть, $\lambda_{B}\left(b_{1}, x\right) \neq \lambda_{B}\left(b_{2}, x\right)$. Заметим, что граф $G(W)$ есть в точности ассоциированный граф из теоремы 3.1 .

Пусть $M=U$ и $n=r$. Тогда справедлива следующая теорема.

Теорема 5.22. $W$ является контрольным экспериментом для $A$ и $\mathbf{A}(U, M)$ тогда и только тогда, когда выполняются следующие условия: 
(1) $W \subseteq L_{A}$;

(2) $\Phi_{B}^{1}=\Phi_{A}^{1}$, где $B=[D(W)]_{U}$;

(3) граф $G(W)$ однозначно раскрашиваем.

Пусть теперь $M=U$ и $n<r$. Тогда для каждого $x$ найдется $y$ такой, что $(x, y) \notin \Phi_{A}^{1}$. Легко привести пример, когда в этом случае условий 1-3 недостаточно для того, чтобы $W$ был контрольным для $A$ и $\mathbf{A}(U, M)$.

Теорема 5.23. $W$ является контрольным для $A$ и $\mathbf{A}(U, M)$ тогда и только тогда, когда $[D(W)]_{U}=A$.

Рассмотрим условия существования и сложность простого, то есть состоящего из одного слова, контрольного эксперимента.

Теорема 5.24. Для $A \in \mathbf{A}(U)-\mathbf{C}(U, M)$ и непустого $\mathbf{C}(U, M)$ существует простой контрольный эксперимент длины, не превосходящей $n$.

Перейдем к рассмотрению условий существования простого контрольного эксперимента для $A \in \mathbf{A}(U, M)$ и $\mathbf{A}(U, M)$. Слоем автомата $A$ называется максимальное по включению подмножество $B$ состояний, в котором для любых $a, b \in B$ aw $=b$ для некоторого $w \in L_{a}$. Слой $B$ называется нетривиальным, если это слово не пусто для некоторых $a, b \in B$. Ясно, что слои образуют разбиение множества состояний автомата. Автомат назовем правильным, если существует последовательность $B_{0}, \ldots, B_{k}$ всех слоев, для которой одновременно выполняются следующие условия.

(1) Из $i$-го слоя, $0 \leqslant i<k$, исходит ровно одна дуга $\left(b_{i}, u_{i}, a_{i}+1\right)$, оканчивающаяся вне него, в следующем слое; все дуги, начинающиеся в $k$-ом слое, оканчиваются в нем.

(2) Если в состоянии $a \in B_{i}, 0 \leqslant i \leqslant k$, оканчивается больше одной дуги эталона, то существует дуга $(a, u, b)$, для которой $u \in M$ и $b \in B_{i}$.

(3) Во всяком цикле каждого слоя существует отмеченное состояние $a$, порождающее некоторый маркер $u$, причем состояние принадлежит этому слою.

В правильном эталоне состояние $a_{i}$ называется начальным, а состояние $b_{i}-$ конечным состоянием $i$-го слоя, $0 \leqslant i \leqslant k$. Заметим, что начальным состоянием слоя $B_{0}$ является начальное состояние $a_{0}$ эталона. Последний слой $B_{k}$ не имеет, по условию 1 , конечного состояния. По условию 3 , правильный эталон принадлежит классу $\mathbf{B}(U, M)$. Известно (см., например, теорему 2.1 на с. 51 в [4]), что условие 1 равносильно существованию обхода, то есть пути из начального состояния автомата, который переходит через все его дуги. При $m \geqslant 2$ все слои правильного автомата являются нетривиальными, и, в силу условия 3, правильный автомат содержит маркеры. Поэтому число слоев правильного автомата не превосходит числа маркеров и тем самым не превосходит $m r$.

Теорема 5.25. Простой контрольный эксперимент относительно $A \in \mathbf{A}(U, M) u$ $\mathbf{A}(U, M)$ сущеествует тогда и только тогда, когда А правилен.

Рассмотрим теперь распознающие эксперименты в финитно-определенных классах второго рода. 
Теорема 5.26. (1) Все классы $F, F \subset \mathbf{B}(U, M)$ являются финитно-распознаваемыми.

(2) Пусть $M$ не пусто. Все классы $F, \mathbf{B}(U, M) \subseteq F \subseteq \mathbf{A}(U, M)$ не являются финитнораспознаваемыми.

Предложение 5.9. Пусть $M$ не пусто. Класс $F=\mathbf{A}(U, M)$ финитно-распознаваем точно тогда, когда он конечен.

Теорема 5.26 и предложение 5.9 показывают, что $\mathbf{B}(U, M)$ является максимальным финитно-распознаваемым подклассом класса $\mathbf{A}(U, M)$.

Рассмотрим условия существования алгоритмов распознавания для других подклассов, порожденных маркерами. Из теоремы 2.1 в [58] и теорем о существовании контрольных экспериментов в финитно-определенных классах непосредственно вытекают следующие утверждения.

Следствие 5.7. Пусть $M$ не пусто. Тогда класс $\overline{\mathbf{A}}(U, M)$ не является финитно-распознаваемилм.

Следствие 5.8. Если класс $\mathbf{C}(U, M)$ состоит из автоматов, не имеющих отмеченных состояний и содержит больше одного автомата, то этот класс не является финитнораспознаваемым.

Пусть $M_{x}$ непусто. Зафиксируем $x \in X$ и натуральное $k>1$. Рассмотрим подкласс $\mathbf{B}(U, x, k) \subseteq \mathbf{A}(U)-\mathbf{C}(U, M)$ всех таких автоматов $A$, для которых одновременно выполняются следующие условия.

(1) Все состояния $a \in A$ достижимы по $x$, то есть $a=\delta\left(a_{0}, x^{i}\right)$ для некоторого $i$.

(2) В единственном цикле по $x$ существует отмеченное состояние, то есть если $\delta\left(a, x^{i}\right)=a$, то состояние отмечено для некоторого $j, 0 \leqslant j \leqslant i$.

(3) Любые разные и отмеченные одним и тем же маркером состояния $k$-отличимы.

Можно показать, что для всех $r \geqslant 2, x \in X$ и $k \geqslant 2$ класс $\mathbf{B}(U, x, k)$ может быть бесконечным.

Пусть

$$
\begin{aligned}
& \overline{\mathbf{A}}(U, x, k)=\overline{\mathbf{A}}(U, M) \cap \mathbf{B}(U, x, k), \\
& \mathbf{A}(U, x, k)=\mathbf{A}(U, M) \cap \mathbf{B}(U, x, k), \quad k \geqslant 2 .
\end{aligned}
$$

Теорема 5.27. Класс $\mathbf{B}(U, x, k)$ бесконечен тогда и только тогда, когда бесконечен класс $\mathbf{A}(U)-\mathbf{C}(U, M)$ и $M_{x}$ не nусm.

Теорема 5.28. Т4.50 Любой класс $F \subseteq \mathbf{B}(U, x, k)$ является финитно-распознаваемыл.

Следствие 5.9. Всякий класс $F \subseteq \overline{\mathbf{A}}(U, x, k) \cup \mathbf{B}(U, M)$ является финитно-распознаваемым. 


\section{Список литературы}

1. Кон П., Универсальная алгебра. Мир, Москва, 1968.

2. Брауэр Р., Введение в теорию конечных автоматов. Радио и связь, Москва, 1987.

3. Богомолов А. М., Барашко А. С., Грунский И. С., Эксперименты с автоматами. Наукова думка, Киев, 1973.

4. Богомолов А. М., Грунский И. С., Сперанский Д. В., Контроль и преобразования дискретных автоматов. Наукова думка, Киев, 1975.

5. Богомолов А. М., Салий В. Н., Алгебраические основы теории дискретных систем. Наука, Москва, 1997.

6. Грунский И. С., Козловский В. А., Пономаренко Г. Г., Представления конечных автоматов фрагментами поведения. Наукова думка, Киев, 1990.

7. Кудрявцев В. Б., Алешин С. В., Подколзин А. С., Введение в теорию автоматов. Наука, Москва, 1985.

8. Трахтенброт Б. А., Барздинь Я. М., Конечные автоматы (поведение и синтез). Наука, Москва, 1970.

9. Харари Ф., Теория графов. Мир, Москва, 1973.

10. Ахо А., Хопкрофт Дж., Ульман Дж., Построение и анализ вычислительных алгоритмов. Мир, Москва, 1979.

11. Грунский И. С., Козловский В. А., Синтез и идентификация автоматов. Наукова думка, Киев, 2004.

12. Гилл А., Введение в теорию конечных автоматов. Наука, Москва, 1966.

13. Глушков В. М., Абстрактная теория автоматов. Успехи матем. наук (1961) 16, №5 (101), 3-62.

14. Мур Э. Ф., Умозрительные эксперименты с последовательностными машинами. В сб.: Автоматы. ИЛ, Москва, 1956, с. 179-210.

15. Bhattacharyya A., Checking experiments on sequential machines. Wiley, New Delhi, 1989.

16. Kohavi Z., Switching and finite automata theory. McGraw-Hill, New York, 1970.

17. Максименко И. К., Эксперименты в финитно-определенных метрических пространствах автоматов, Автореферат канд. дисс. физ.-мат. наук. Саратовский госуниверситет, Саратов, 2000.

18. Богомолов С. А., О восстановлении автоматов по их следам, Автореферат канд. дисс. физ.-мат. наук. ВЦ АН СССР, Москва, 1986.

19. Спивак М. А., К синтезу конечного автомата по его множеству экспериментов. Кибернетика (1969) 5, 15-20.

20. Буевич В. А., Каландаришвили Н. Г., Таль А. А., Об описании конечного автомата с помощью конечного множества вход-выходных слов. Автоматика и телемеханика (1970) 1, 112-122.

21. Иванов Н. Н., Михайлов Г. И., Руднев В. В., Таль А. А., Конечные автоматы - эквивалентность и поведение. Наука, Москва, 1984.

22. Грунский И. С., Анализ поведения конечных автоматов. ЛГПУ, Луганск, 2003.

23. Евтушенко Н. В., Лебедев А. В., Петренко А. Ф., О проверяющих экспериментах с недетерминированными автоматами. Автоматика и вычислительная техника (1991) 6, 81-85.

24. Лукьянов Б. Д., О различающих и контрольных экспериментах с недетерминированными автоматами. Кибернетика и системный анализ (1995) 31, 69-76.

25. Лукьянов Б. Д., Детерминированные реализации недетерминированных автоматов. Кибернетика и системный анализ (1996) 32, 34-50.

26. Скорняков Л. А., Общая алгебра, 1. Наука, Москва, 1990. 
27. Петренко А. Ф., Автоматные методы анализа совместимости средств взаимодействия 6 открытых сетях, Автореферат докт. дисс. техн. наук. ИЭП Латвийской ССР, Рига, 1988.

28. Петренко А. Ф., Эксперименты над протокольными объектами. Автоматика и вычислительная техника (1987) 1, 16-21.

29. Petrenko A. F., Yevtushenko N., Dssouli R., Grey-box FSM-based testing strategies, Dept. Publ. 991. Dept. IRO, Univ. de Montreal, 1994.

30. Захаров В. Н., Поспелов Д. А., Хазацкий В. Е., Системы управления. Задание. Проектирование. Реализация. Энергия, Москва, 1972.

31. Крывый С. А., Матвеева Л. Е., Формальные методы анализа свойств систем. Кибернетика $u$ системный анализ (2003) 39, 15-36.

32. Пархоменко П. П., Основы технической диагностики. Энергия, Москва, 1976.

33. Fault-tolerant computing: Theory and techniques, 1 (Pradhan D. K., ed.). Prentice Hall, Upper Saddle River, NJ, 1986.

34. Kornoushenko E. K., Monitoring of logic-dynamic systems based on sampled performance data. $J$. Comput. Syst. Sci. Int. (1992) 30, №6, 107-117.

35. Hennie F. C., Fault detecting experiments for sequential circuits. In: Proc. 5th Annual Symp. on Switching Circuit Theory and Logical Design. Princeton Univ., Princeton, NJ, 1964, pp. 95-110.

36. Василевский М. П., О распознавании неисправностей автомата. Кибернетика (1973) 4, 93-108.

37. Бородай С. Ю., Эксперименты в эффективно заданных классах автоматов, Автореферат канд. дисс. физ.-мат. наук. СГУ, Саратов, 1997.

38. Бородай С. Ю., Условные и безусловные эксперименты с классами реализаций НД-автоматов. В сб.: Тезисы докл. ХІ Межд. конф. по проблемам теор. кибернетики. СВНЦ, Ульяновск, 1996, c. $27-28$.

39. Кузнецов А. Б., Трахтенброт Б. А., Исследование частично рекурсивных операторов средствами теории бэровского пространства. Доклады АН СССР (1955) 105, №5, 897-900.

40. Максименко И. И., Распознавание в эффективно-заданных классах автоматов. Труды ИПММ НАН Украинь (1998) 2, 115-123.

41. Корноушенко Е. К., Диагностирование дискретных динамических систем по накопленной информации, Автореферат докт. дисс. техн. наук. ИПУ, Москва, 1992.

42. Козловский В. А., О распознавании автомата относительно локально порожденного класса. Доклады АН СССР (1981) 285, №5, 1047-1049.

43. Козловский В. А., О распознавании локальных неисправностей автомата, Автореферат канд. дисс. физ.-мат. наук. ВЦ АН СССР, Москва, 1981.

44. Козловский В. А., Локальные неисправности автомата и их обнаружение. Матем. вопросы киберн. (1991) 3, 167-186.

45. Козловский В. А., Копытова О. М., Представления автоматов относительно $m$-плотных классов. В сб.: Материаль VIII Межд. Семинара “Дискретная математика и ее приложения”. МГУ, Москва, 2004, с. 277-280.

46. Козловский В. А., О представлениях групповых автоматов. Кибернетика и системный анализ (1996) 32, 21-28.

47. Козловский В. А., О структуре контрольных экспериментов с автоматом. Кибернетика (1978) 3, 19-33.

48. Kozlovskii V. A., On the complexity of analyzing experiments for checking local faults of an automaton. Lecture Notes Computer Sci. (1987) 278, 259-262.

49. Грунский И. С., Козловский В. А., Копытова О. М., Представления автоматов и анализ атак на криптосистемы. Искусственный интеллект (2004) 4, 764-775. 
50. Евтушенко Н. В., Матросова А. Ю., К синтезу контролепригодных автоматных сетей. Техническая диагностика (1991) 3, 143-152.

51. Евтушенко Н. В., Петренко А. Ф., Метод построения проверяющих экспериментов для произвольного недетерминированного автомата. Автоматика и вычислительная техника (1990) 5 , $73-76$.

52. Евтушенко Н. В., Петренко А. Ф., О проверяющих возможностях кратных экспериментов. Автоматика и вычислительная техника (1989) 3, 9-14.

53. Максименко И. И., Эксперименты в классе реализаций недетерминированных автоматов. Доклады НАН Украины (1999) 7, 95-99.

54. Грунский И. С., Идентификация управляющих систем автоматного типа. В сб.: Труды ICIM'98. ТГПИ, Таганрог, 1998, с. 107-109.

55. Грунский И. С., Максименко И. И., О распознавании детерминированных автоматов, задаваемых НД-автоматами. В сб.: Труды III Межд. Конф. “Дискретные модели в теории управляющих систем”, Красновидово. МГУ, Москва, 1998, с. 23-29.

56. Грунский И. С., Максименко И. И., Эксперименты с маркированными автоматами, Препринт 96.02. ИПММ НАН Украины, Донецк, 1998.

57. Грунский И. С., Максименко И. И., Об экспериментах с автоматами при отсутствии верхней оценки числа состояний. Кибернетика и системный анализ (1999) 4, 59-71.

58. В. Б. Кудрявцев, И. С. Грунский, В. А. Козловский, Анализ поведения автоматов. Дискретная математика (2009) 21, №1, 3-35.

Статья поступила 20.06.2008. 